\title{
ON STRONG AFFINE REPRESENTATIONS OF THE POLYCYCLIC MONOIDS
}

\author{
MIKLÓS HARTMANN AND TAMÁS WALDHAUSER
}

\begin{abstract}
Jones and Lawson have discovered in 16 that certain representations of the so-called polycyclic monoids are closely related to some permutative representations of the Cuntz algebras $\mathcal{O}_{n}$ studied by Bratteli and Jorgensen in 4]. We investigate these representations of the polycyclic monoids, and we generalize some results from [16]. We give a (sharp) upper bound on the number of atoms in case one of the parameters tends to infinity and present an infinite family of representations having only one atom. Furthermore, by making use of a $\mathrm{C}++$ program we present some observations regarding the number of atoms in the case $n=3$.
\end{abstract}

\section{INTRODUCTION}

Let $d_{1}, \ldots, d_{n}$ be a complete system of residues modulo $n$ (i.e., integers that represent each residue class modulo $n$ ) and let $f_{i}: \mathbb{Z} \rightarrow n \mathbb{Z}+d_{i}, x \mapsto n x+d_{i}$. The union of the inverse maps $f_{i}^{-1}$ constitutes a transformation $R: \mathbb{Z} \rightarrow \mathbb{Z}$ defined by $R(x)=\frac{x-d_{i}}{n}$, where $d_{i}$ is the unique element of the set $D=\left\{d_{1}, \ldots, d_{n}\right\}$ such that $x \equiv d_{i}(\bmod n)$. In this paper we investigate the set $B_{\infty}(D)$ of periodic points of $R$ :

$$
B_{\infty}(D):=\left\{x \in \mathbb{Z}: R^{\ell}(x)=x \text { for some } \ell \in \mathbb{N}\right\} .
$$

Determining the number and structure of periodic orbits of $R$ is a highly nontrivial problem, which is of interest on its own, and it is also connected to the study of representations of the polycyclic monoids and of the Cuntz $C^{*}$-algebras as well as to generalized radix representations and the corresponding "digit tilings" of Euclidean spaces. We briefly describe these connections below, but in the rest of the paper we refer only to the elementary setup outlined above, in order to make the paper more accessible and self-contained.

1.1. Radix representations. Iterating the map $R$ on a given integer $x$ and recording at each step the element of $D$ that we used, we obtain a sequence $a_{0}, a_{1}, \ldots \in D$ such that $R^{i}(x) \equiv a_{i}(\bmod n)$ for every $i \in \mathbb{N}_{0}$. It is then straightforward to verify that $x=$ $a_{0}+n a_{1}+\cdots+n^{k-1} a_{k-1}+n^{k} R^{k}(x)$ for all $k \in \mathbb{N}$. If $x \geq 0$ and $D=\{0,1, \ldots, n-1\}$ is the canonical residue system modulo $n$, then $R^{k}(x)=0$ for sufficiently large $k$, hence we get $x=a_{0}+n a_{1}+\cdots+n^{k-1} a_{k-1}$, which is just the usual base- $n$ representation of $x$. For other choices of $D$ and $x$, we may not have a finite representation, but we can still regard the formal infinite sum $x=a_{0}+n a_{1}+\cdots+n^{k-1} a_{k-1}+\ldots$ as a generalized $n$-ary representation of $x$ corresponding to the digit set $D$. It turns out that the sequence of digits $a_{0}, a_{1}, \ldots$ is always ultimately periodic, and then one can interpret the infinite radix representation as a geometric series (see Remark 2.10).

Several authors have studied similar, and even more general radix representations: Kátai and Szabó [18] considered number systems for Gaussian integers; Gilbert generalized their results to quadratic number fields [11] and also investigated radix representations in arbitrary algebraic number fields [10. Another widely investigated generalization concerns base- $N$ representations of elements of $\mathbb{Z}^{\nu}$, where $N$ is a $\nu \times \nu$

2010 Mathematics Subject Classification. 20M18, 20M30, 46L05, 11A63, 37P35.

Key words and phrases. Polycyclic monoids; Cuntz inverse semigroups; affine representations; Cuntz algebras; permutative representations; atoms of representations; branching function systems; discrete dynamical systems; periodic points; Cantor set; fractals; radix representations; self-affine tiles. 
expanding integer matrix (i.e., every eigenvalue of $N$ has absolute value greater than one) and the set $D$ of "digits" is a system of representatives of the cosets corresponding to the subgroup $N \mathbb{Z}^{\nu}$ of $\mathbb{Z}^{\nu}$. We refer the reader to the survey paper by Vince [30] and the references therein for more information on this topic.

1.2. Fractals, tilings and wavelets. Radix representations are closely related to tilings and self-affine sets, which in turn have applications in the theory of wavelets. First, let us consider once more the standard $n$-ary number system with digit set $D=\{0,1, \ldots, n-1\}$, this time considering $x \in \mathbb{R}$ instead of $x \in \mathbb{Z}$. If $x \geq 0$, then $x$ has a (possibly infinite and possibly not unique) base- $n$ expansion $x=n^{k} a_{k}+\cdots+$ $a_{0}+n^{-1} a_{-1}+\ldots$ with $a_{i} \in D$. Numbers having no digits to the left of the "decimal" point (i.e., $a_{i}=0$ for all $i \geq 0$ ) form the interval [0,1], whereas numbers with no digits to the right of the "decimal" point (i.e., $a_{i}=0$ for all $i<0$ ) constitute the set $\mathbb{N}_{0}$ of nonnegative integers. The subgroup of $\mathbb{R}$ generated by $\mathbb{N}_{0}$ is the lattice $\mathbb{Z}$, and it is clear that $\mathbb{R}$ is the union of translates of $[0,1]$ by elements of $\mathbb{Z}$; moreover, any two of these translated intervals intersect only in a set of measure zero (in fact, in at most one point), i.e., $[0 ; 1]$ tiles $\mathbb{R}$.

Now if $D=\left\{d_{1}, \ldots, d_{n}\right\}$ is an arbitrary complete system of residues modulo $n$, then the interval $[0,1]$ shall be replaced by the set

$$
\mathbb{T}(D):=\left\{\sum_{i=1}^{\infty} n^{-i} a_{i}: a_{i} \in D\right\} .
$$

(This set depends on $D$ as well as on $n$, but $n$ shall always be clear from the context, and we will sometimes also omit $D$ from the notation when there is no risk of ambiguity.) Note that $\mathbb{T}$ is a self-affine set (a union of smaller copies of itself):

$$
\mathbb{T}=\frac{d_{1}}{n}+\frac{1}{n} \cdot \mathbb{T} \cup \cdots \cup \frac{d_{n}}{n}+\frac{1}{n} \cdot \mathbb{T} .
$$

Bandt [2] proved that $\mathbb{T}$ is a compact set with nonempty interior, and Lagarias and Wang 24] showed that the boundary of $\mathbb{T}$ has measure zero. This implies that $\mathbb{T}$ is Jordan measurable, and Gröchening and Haas [13] determined its (Jordan or Lebesgue) measure:

$$
\mu(\mathbb{T})=\operatorname{gcd}\left\{d_{i}-d_{j}: 1 \leq i, j \leq n\right\} .
$$

Again, $\mathbb{T}$ yields a tiling of $\mathbb{R}$ (hence the notation $\mathbb{T}$ ), but this time we need to use translates by the lattice $\mu(\mathbb{T}) \cdot \mathbb{Z}$ (translates by $\mathbb{Z}$ would give a $\mu(\mathbb{T})$-fold covering of R) $[13,22,24$.

Similar tiling phenomena occur in the $\nu$-dimensional case outlined in the previous subsection (see, e.g., 23, 22, 24, 30]); in this case we do not have a simple formula for the measure of the corresponding tile $\mathbb{T}$, but Bondarenko and Kravchenko provided an algorithm to compute $\mu(\mathbb{T})[3$. The tile $\mathbb{T}$ is again a self-affine set, often with a fractal structure, and its topological and geometrical properties have been studied by many authors 1, 2, 8, 14, 20. Gröchenig and Madych [12 established a one-to-one correspondence between certain wavelet bases of $L^{2}\left(\mathbb{R}^{\nu}\right)$ and tiles $\mathbb{T}$ of $\mathbb{R}^{\nu}$ of measure one. Further results relating tilings and wavelets can be found in [7, 21].

1.3. Operator algebras. The Cuntz $C^{*}$-algebra $\mathcal{O}_{n}$ is the $C^{*}$-algebra generated by $n$ pairwise orthogonal isometries on a Hilbert space. More precisely, let $\mathcal{H}$ be an infinite-dimensional separable Hilbert space, and let $S_{i}(i=1, \ldots, n)$ be bounded linear operators on $\mathcal{H}$ such that $S_{i}^{*} S_{i}=I$ for every $i$ and $S_{1} S_{1}^{*}+\cdots+S_{n} S_{n}^{*}=I$. This implies that $S_{i}^{*} S_{j}=0$ whenever $i \neq j$, and Cuntz [5] has proven that the $C^{*}$ algebra $\mathcal{O}_{n}$ generated by $S_{1}, \ldots, S_{n}$ is (up to isomorphism) independent of the choice of these isometries - hence the definite article in the first sentence of this paragraph. The so-called permutative representations of $\mathcal{O}_{n}$ are defined by $S_{i} e_{j}=e_{f_{i}(j)}$, where $\left\{e_{j}: j \in \mathbb{Z}\right\}$ is an orthonormal basis of $\mathcal{H}$. Thus, in this case the isometries $S_{i}$ permute the elements of the orthonormal basis. These operators satisfy the defining relations of $\mathcal{O}_{n}$ if and only if each $f_{i}$ is injective and their ranges form a partition of $\mathbb{Z}$. In 
particular, the maps $f_{i}(x)=n x+d_{i}(i=1, \ldots, n)$ yield permutative representations of $\mathcal{O}_{n}$ when $d_{1}, \ldots, d_{n}$ be a complete system of residues modulo $n$. Bratteli and Jorgensen [4] have studied such representations and their relationship to the dynamics of the map $R$ and to the structure of the tile $\mathbb{T}$, also in the $\nu$-dimensional case mentioned in Subsection 1.1 (we borrow some notation from this paper, in particular, the symbol $B_{\infty}$ for the set of periodic points). Let us recall a proposition from [4 that will play a crucial rule in Section 6 .

Proposition 1.1 (4). If $D$ is a complete system of residues modulo $n$, then the negatives of the integers in $\mathbb{T}(D)$ are exactly the periodic points of $R: B_{\infty}(D)=$ $-\mathbb{T}(D) \cap \mathbb{Z}$.

Jeong [15] generalized results of [4] about irreducible subrepresentations of permutative representations and obtained an upper bound for the number of periodic points as well as a sufficient condition for $R$ to have only one periodic point. Continued fractions can be regarded as a "base- $\infty$ " number system, where the set of digits is $\mathbb{N}$. Kawamura, Hayashi and Lascu [19] adopted this point of view to explore a connection between continued fractions and permutative representations of $\mathcal{O}_{\infty}$; here quadratic irrationals correspond to periodic points.

1.4. Semigroups. For each $n \geq 2$ the polycyclic monoid $\mathcal{P}_{n}$ is defined as a monoid with zero by the presentation

$$
\mathcal{P}_{n}=\left\langle a_{1}, \ldots, a_{n}, a_{1}^{-1}, \ldots, a_{n}^{-1}: a_{i}^{-1} a_{i}=1 \text { and } a_{i}^{-1} a_{j}=0, i \neq j\right\rangle .
$$

These monoids were introduced by Nivat and Perrot in [29] and have been rediscovered by Cuntz [5], hence they are sometimes referred to as the Cuntz inverse semigroups. Besides the Cuntz algebras, the monoids $\mathcal{P}_{n}$ are also related to the Thompson groups $V_{n, 1}$ [25, 26] and pushdown automata [17]. The important role played by these monoids within semigroup theory is underlined by a result of Meakin and Sapir [28] showing that congruences of free monoids correspond to certain submonoids of polycyclic monoids (this was generalized to right congruences in [27]), which allows one to translate general questions about monoids to questions about submonoids of polycyclic monoids.

A representation of $\mathcal{P}_{n}$ is a monoid homomorphism from $\mathcal{P}_{n}$ to a symmetric inverse monoid, i.e., to the monoid of all partial bijections on a set $X$. All such representations can be constructed in the following way. Let $X$ be an infinite set and let $X_{1}, \ldots, X_{n}, Y$ be disjoint subsets of $X$ such that their union is $X$ and the subsets $X_{i}$ have the same cardinality as $X$. For every $1 \leq i \leq n$, let $f_{i}: X \rightarrow X_{i}$ be a bijection which we will consider to be a partial bijection on $X$. In this case we can define a representation of $\mathcal{P}_{n}$ by sending $a_{i}$ to $f_{i}$ and $a_{i}^{-1}$ to $f_{i}^{-1}$. If $Y=\emptyset$ then we say that the representation is strong [26]. Thus a strong representation can be given by the data $\left(X ; f_{1}, \ldots, f_{n}\right)$, which is called a branching function system [4. The strong representations given by $\left(X ; f_{1}, \ldots, f_{n}\right)$ and $\left(\tilde{X} ; \tilde{f}_{1}, \ldots, \tilde{f}_{n}\right)$ are equivalent if there is a bijection $\varphi: X \rightarrow \tilde{X}$ such that $f_{i} \varphi=\varphi \tilde{f}_{i}$ for $i=1, \ldots, n$.

Lawson [27] described representations of the polycyclic monoids in terms of strong ones, hence it suffices to study strong representations. Affine representations constitute an important class of strong representations. These are given by $X=\mathbb{Z}^{\nu}$ and $f_{i}(\mathbf{x})=N \mathbf{x}+\mathbf{d}_{i}$, where $N$ is a nonsingular $\nu \times \nu$ matrix over $\mathbb{Z}$ and $\mathbf{d}_{1}, \ldots, \mathbf{d}_{n}$ is a system of representatives of the cosets corresponding to the subgroup $N \mathbb{Z}^{\nu}$ of $\mathbb{Z}^{\nu}$ (cf. Subsections 1.1 and 1.2 . Here $n=\left[\mathbb{Z}^{\nu}: N \mathbb{Z}^{\nu}\right]$ equals the determinant of $N$.

In this paper we consider only one-dimensional affine representations, i.e., the case $\nu=1$. As we shall see, even this seemingly simple case raises highly nontrivial problems, and we are still very far from a full understanding of the one-dimensional strong affine representations of $\mathcal{P}_{n}$. In the remainder of this section we review some results from the literature that are specific to this one-dimensional case, and we briefly and informally explain our contributions to this topic. 
1.5. Main results. The structure of the one-dimensional strong affine representation of $\mathcal{P}_{n}$ corresponding to $\left(\mathbb{Z} ; f_{1}, \ldots, f_{n}\right)$ with $f_{i}(x)=n x+d_{i}$, where $D=\left\{d_{1}, \ldots, d_{n}\right\}$ is a complete system of residues modulo $n$, is to a large extent determined by the set $B_{\infty}(D)$ of periodic points (see Section 2 for more details). Bandt 2 already mentions that $R$ has only a finite number of periodic orbits and hints at the importance of studying these orbits in connection with radix representations and tilings, and several authors considered sporadic examples, mainly for $n=3$ (see, e.g., [4, 12, 30]). Let us also mention the recent paper by Dutkay and Haussermann [6] where the case $n=4, D=\{0, m\}$ with $m$ being an odd integer is analyzed. Clearly, here $D$ is not a complete system of residues modulo $n$ (hence the map $R$ is only partially defined), but the periodic orbits of $R$ still have interesting number-theoretic properties and they have relevance to the harmonic analysis of a certain fractal measure.

Our work is motivated by the papers [4] and [16], which appear to contain the only systematic studies of the periodic points, namely, for $n=2$. In this case one can assume without loss of generality that $D=\{0, p\}$, where $p$ is an odd positive integer (see Fact 2.2). Bratteli and Jorgensen [4 proved that $B_{\infty}(0, p)=\{-p, \ldots,-1,0\}$ and that the period of an arbitrary $x \in B_{\infty}(0, p)$ equals the order of 2 modulo $p / \operatorname{gcd}(x, p)$. It is well known (and a nice exercise in number theory) that this order is the same as the period of the binary expansion of the fraction $x / p$. Jones and Lawson [16] showed that this is not a coincidence: the structure of the cycle containing $x$ is strongly related to the digits in the binary expansion of $x / p$. Two other special cases are also considered in [4, namely, $B_{\infty}(0,1, \ldots, n-1)=\{-1,0\}$ and $B_{\infty}(1,3,5)=\{-2,-1\}$.

In all of the special cases mentioned above, $d_{1}, \ldots, d_{n}$ is an arithmetic sequence. In Section 3 we generalize the results of [4, 16] by proving that the set $B_{\infty}(D)$ consists of the integers in the interval $\mathcal{I}:=\left[-\frac{d_{n}}{n-1},-\frac{d_{1}}{n-1}\right]$ whenever $d_{1}, \ldots, d_{n}$ is an arithmetic sequence (Theorem 3.1), and we also relate the structure of the cycle containing the integer $x \in B_{\infty}(D)$ to the $n$-ary expansion of the fraction $\frac{(n-1) x+d_{1}}{d_{n}-d_{1}}$ (Theorem 3.3. It is easy to see that we have $B_{\infty}(D) \subseteq \mathcal{I}$ for every $D$ (Fact 2.4), hence we can say that for arithmetic sequences the set of periodic points is as large as possible. We show in Section 4 that we may have such a large set of periodic points even if $d_{1}, \ldots, d_{n}$ is not an arithmetic sequence. We will characterize explicitly the sequences satisfying $B_{\infty}(D)=\mathcal{I} \cap \mathbb{Z}$ (Theorem 4.1), and we will see that these sequences are in some sense not far from being arithmetic (Remark 4.2). In Section 6 we study the asymptotic behaviour of the number of periodic points as one of the parameters, say $d_{n}$, tends to infinity, while $d_{1}, \ldots, d_{n-1}$ are fixed. We prove that $\left|B_{\infty}(D)\right|=O\left(d_{n}^{\log _{n} 2}\right)$ (Theorem 6.4), and we also provide infinite series of examples showing that this upper bound cannot be improved (Theorem 6.5). For a lower bound, we have the trivial estimate $\left|B_{\infty}(D)\right| \geq 1$, and we will prove in Section 5 that in general one cannot have a better lower bound, since it is possible to let $d_{n}$ tend to infinity in such a way that the number of periodic points stays constant 1 (Theorem 5.1).

\section{Preliminaries}

We recall some facts from [4, 16, 27] that we shall need in the sequel; we present these in terms of branching functions systems, which provide a combinatorial framework for studying permutative representations of the Cuntz $C^{*}$-algebras $\mathcal{O}_{n}$ and strong representations of the polycyclic monoids $\mathcal{P}_{n}$, hence familiarity with the theory of operator algebras or semigroups is not assumed.

A branching function system is a tuple $\left(X ; f_{1}, \ldots, f_{n}\right)$, where $X$ is an infinite set and $f_{i}: X \rightarrow X(i=1, \ldots, n)$ are injective maps such that their ranges form a partition of $X$. One can visualize a branching function system as a directed graph with colored edges: the vertices are the elements of $X$, and an arrow of color $i$ is drawn from $x$ to $y$ if $f_{i}(x)=y$; we shall frequently refer to this graph in the following. We say that two branching function systems are equivalent if the corresponding colored graphs are isomorphic (where the isomorphism is required to preserve colors); this corresponds to the usual notion of equivalence of representations of semigroups. 

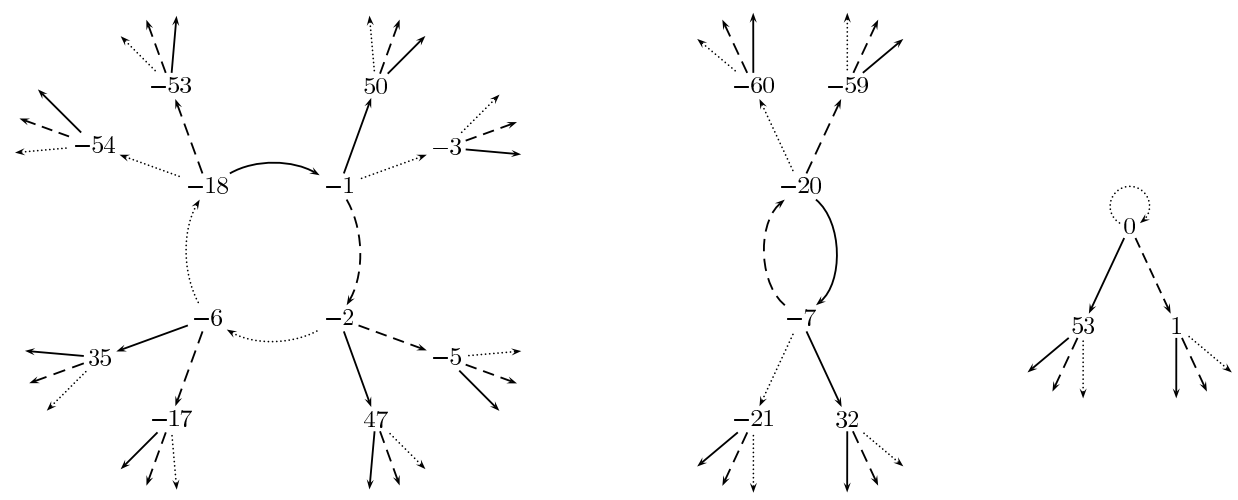

FIGURE 1. A part of the graph of the branching function system with parameters $\left(d_{1}, d_{2}, d_{3}\right)=(0,1,53)$

Observe that the definition of a branching function system requires that for every vertex there are $n$ outgoing edges (one of each of the $n$ colors, leading to $n$ different vertices) and that there is exactly one incoming edge. These two properties imply that there can be at most one cycle in every connected component of the graph and this cycle must be directed. (By a cycle we mean a closed path, as usual in graph theory. However, note that this terminology is different from that of 4, 16; see Remark 2.1]) It is straightforward to verify that if a connected component contains a cycle, then the order of the colors appearing in the cycle determine that connected component up to isomorphism. (See Figure 1 it shows only a finite part of the graph of a branching function system with $n=3$, but the missing parts are easy to imagine: an infinite ternary tree is rooted at the vertices $-54,-53,50,-3$, etc. The numbering of the vertices will be explained later.) Let us note that cycle-free components can be more complicated; however, for the special branching function systems that we consider in this paper, each component contains a cycle (see Fact 2.4).

The inverses of the maps $f_{i}$ are partial bijections on $X$ whose union is a surjective map $R: X \rightarrow X$, where $R(x)$ is the unique element $y \in X$ such that there exists $i \in$ $\{1, \ldots, n\}$ with $f_{i}(y)=x$. The branching function system $\left(X ; f_{1}, \ldots, f_{n}\right)$ can also be studied via the discrete dynamical system $(X ; R)$. The trajectory $x, R(x), R^{2}(x), \ldots$ of a point $x$ can be seen as a walk in the graph that starts from $x$ and follows the arrows backwards. Let $\sigma(x)=\left(j_{0}, j_{1}, \ldots\right)$ be the sequence of the colors of the edges in this walk: $x=f_{j_{0}}(R(x)), R(x)=f_{j_{1}}\left(R^{2}(x)\right)$, etc.; this defines the so-called coding map $\sigma: X \rightarrow\{1, \ldots, n\}^{\mathbb{N}_{0}}$. We say that $x$ is a periodic point if the trajectory of $x$ is periodic, i.e., if $x$ is a fixed point of $R^{\ell}(x)$ for some $\ell \in \mathbb{N}$. If $\ell$ is the least positive exponent such that $R^{\ell}(x)=x$, then (and only then) $x$ lies on a cycle of length $\ell$ in the graph. Following [4, we denote the set of periodic points by $B_{\infty}$. Using the terminology of discrete dynamical systems, we shall say that a set $A \subseteq X$ is positively invariant if $R(A) \subseteq A$, and $A$ is said to be absorbing if for every $x \in X$, the trajectory of $x$ lies eventually in $A$, i.e., we have $\left\{R^{t}(x), R^{t+1}(x), \ldots\right\} \subseteq A$ for some $t \in \mathbb{N}$.

Remark 2.1. Let us define two equivalence relations $\sim$ and $\approx$ on $X$ by $x \sim y \Longleftrightarrow$ $\exists s, t: R^{s}(x)=R^{t}(y)$ and $x \approx y \Longleftrightarrow \exists t: R^{t}(x)=R^{t}(y)$. In [4, 16, the main objects of study are the blocks of $\sim$ and $\approx$, which were called there cycles and atoms, respectively. Our terminology is different: we use the term cycle in the graph-theoretical sense, and we refer to the blocks of $\sim$ as connected components. For branching function systems corresponding to one-dimensional affine representations of $\mathcal{P}_{n}$, there is a one-to-one correspondence between atoms and periodic points (see Scholium 3.9 of [4] and Proposition 4.9 of [16]). Since we deal only with this case, we work with the simpler concept of a periodic point instead of that of an atom.

Now let us turn to the special branching function systems mentioned in Subection 1.5. The notation that we introduce here will be used throughout the paper 
without further mention. Let $n \geq 2$ be a positive integer, let $D=\left\{d_{1}, \ldots, d_{n}\right\}$ be a complete system of residues modulo $n$, and let $q_{i}$ and $r_{i}$ denote the quotient and the remainder of $d_{i}$, when divided by $n$, i.e., $d_{i}=n q_{i}+r_{i}$ and $0 \leq r_{i}<n$. Clearly, we have $\left\{r_{1}, \ldots, r_{n}\right\}=\{0, \ldots, n-1\}$. The functions $f_{i}(x):=n x+d_{i}(i=1, \ldots, n)$ define a branching function system $\left(\mathbb{Z} ; f_{1}, \ldots, f_{n}\right)$, and the corresponding dynamical system is $(\mathbb{Z} ; R)$, where $R(x)=\frac{x-d_{i}}{n}$ with $d_{i}$ being the uniqe element of $D$ such that $x \equiv d_{i}(\bmod n)$. As an example, see Figure 1 , which shows the graph of the branching function system corresponding to $n=3$ and $\left(d_{1}, d_{2}, d_{3}\right)=(0,1,53)$, where dotted, dashed and solid arrows represent $f_{1}, f_{2}$ and $f_{3}$, respectively. Iterations of $R$ can be seen in the figure by following the arrows backwards. For instance, the trajectory of 50 is $50,-1,-18,-6,-2,-1,-18,-6,-2, \ldots$ and $\sigma(50)=(3,3,1,1,2,3,1,1,2, \ldots)$.

In the following we collect some basic facts about these branching function systems. These results all appeared in [4, 16, 27; for the reader's convenience we restate and reprove them. Unless otherwise mentioned, we will always assume that $d_{1}<\cdots<d_{n}$.

Fact 2.2. The following sequences give rise to equivalent branching function systems:
a) $d_{1}, \ldots, d_{n}$
b) $-d_{1},-d_{2}, \ldots,-d_{n}$;
c) $d_{1}+k(n-1), d_{2}+k(n-1), \ldots, d_{n}+k(n-1)$, for arbitrary $k \in \mathbb{Z}$.

Proof. It is easy to check that the maps $\beta: \mathbb{Z} \rightarrow \mathbb{Z}, x \mapsto-x$ and $\gamma: \mathbb{Z} \rightarrow \mathbb{Z}, x \mapsto x-k$ establish isomorphisms from the graph corresponding to a) to the graph corresponding to b) and c), respectively.

By this fact, one can always assume that the parameters $d_{1}<\cdots<d_{n}$ are chosen such that $0 \leq d_{1}<n-1$. Let $\mathcal{I}$ denote the interval $\left[-\frac{d_{n}}{n-1},-\frac{d_{1}}{n-1}\right]$, let $\mathcal{A}(D)=\mathcal{I} \cap \mathbb{Z}$ and let $B_{\infty}(D)$ be the set of periodic points, as defined in 1.1 ; sometimes we will write simply $\mathcal{A}$ and $B_{\infty}$ whenever the parameters $d_{1}, \ldots, d_{n}$ are clear from the context. (From Figure 1 we see that $B_{\infty}(0,1,53)=\{-20,-18,-7,-6,-2,-1,0\}$ and we have $\mathcal{A}(0,1,53)=\{-26, \ldots, 0\}$.)

Lemma 2.3. The set $\mathcal{A}$ is a finite positively invariant absorbing set.

Proof. Since $d_{1}<\cdots<d_{n}$, we have $\frac{x-d_{n}}{n} \leq R(x) \leq \frac{x-d_{1}}{n}$ for every $x \in \mathbb{Z}$. From these inequalities one can deduce the following implications:

$$
x<\frac{-d_{n}}{n-1} \quad \Longrightarrow \quad x \quad<R(x)<\frac{-d_{1}}{n-1}
$$

b) $\frac{-d_{n}}{n-1} \leq x \leq \frac{-d_{1}}{n-1} \Longrightarrow \frac{-d_{n}}{n-1} \leq R(x) \leq \frac{-d_{1}}{n-1}$;

c) $\frac{-d_{1}}{n-1}<x \quad \Longrightarrow \frac{-d_{n}}{n-1}<R(x)<\quad x$.

The second implication immediately yields that $\mathcal{A}$ is positively invariant. If $x \notin \mathcal{A}$ then either $x<R(x)<R^{2}(x)<\cdots<R^{t}(x) \in \mathcal{A}$ or $x>R(x)>R^{2}(x)>\cdots>$ $R^{t}(x) \in \mathcal{A}$ for some $t \in \mathbb{N}$ by a) or c) depending on whether $x<-\frac{d_{n}}{n-1}$ or $x>-\frac{d_{1}}{n-1}$. In both cases b) shows that the rest of the trajectory of $x$ stays in $\mathcal{A}$.

Fact 2.4. The graph corresponding to the branching function system determined by $D$ has finitely many connected components, and each component contains a cycle. These cycles are all contained in $\mathcal{A}(D)$, i.e., we have

$$
B_{\infty}(D) \subseteq \mathcal{A}(D) .
$$

The trajectory of every integer $x \in \mathbb{Z}$ is eventually periodic: there exist $\ell, t \in \mathbb{N}$ such that $R^{i+\ell}(x)=R^{i}(x)$ whenever $i \geq t$.

Proof. For every $x \in \mathbb{Z}$, the trajectory of $x$ is finite, since $R^{i}(x)$ belongs to the finite set $\mathcal{A}$ for almost every $i$, by Lemma 2.3 . This implies that every trajectory is eventually periodic, hence each connected component contains a cycle. These cycles are contained in $\mathcal{A}$, thus there are finitely many cycles and finitely many connected components. 
According to Fact 2.4, $B_{\infty}$ is a nonempty set consisting of finitely many cycles, and every trajectory eventually winds around one of these cycles. This implies that the sequence $\sigma(x)$ is also eventually periodic for every $x \in \mathbb{Z}$. We show below how to use these facts to see that $\sigma(x)$ uniquely determines $x$, i.e., that the coding map is injective. (Representations of $\mathcal{O}_{n}$ with an injective coding map are called multiplicityfree representations in [4.)

Fact 2.5. Suppose that the trajectory of $x$ becomes $\ell$-periodic after $t$ terms, i.e., $R^{t+\ell}(x)=R^{t}(x)$, and let $\sigma(x)=\left(j_{0}, j_{1}, \ldots\right)$. Then we have

$$
x=d_{j_{0}}+n d_{j_{1}}+\cdots+n^{t-1} d_{j_{t-1}}-\frac{d_{j_{t}} n^{t}+\cdots+d_{j_{t+\ell-1}} n^{t+\ell-1}}{n^{\ell}-1} .
$$

Proof. From the definition of $R$ and $\sigma$ one can deduce by induction on $k$ that $x=$ $d_{j_{0}}+n d_{j_{1}}+\cdots+n^{k-1} d_{j_{k-1}}+n^{k} R^{k}(x)$ holds for all $k \in \mathbb{N}$. Applying this formula for $k=t$ and $k=t+\ell$ we obtain

$$
\begin{aligned}
x & =d_{j_{0}}+n d_{j_{1}}+\cdots+n^{t-1} d_{j_{t-1}}+n^{t} R^{t}(x) \\
& =d_{j_{0}}+n d_{j_{1}}+\cdots+n^{t-1} d_{j_{t-1}}+n^{t} d_{j_{t}}+\cdots+n^{t+\ell-1} d_{j_{t+\ell-1}}+n^{t+\ell} R^{t+\ell}(x) .
\end{aligned}
$$

By our assumption we have $R^{t+\ell}(x)=R^{t}(x)$, hence $\left(1-n^{\ell}\right) n^{t} R^{t}(x)=n^{t} d_{j_{t}}+\cdots+$ $n^{t+\ell-1} d_{j_{t+\ell-1}}$ follows, and this proves 2.1 .

If $x \in B_{\infty}$ lies on a cycle of length $\ell$, then the sequence $\sigma(x)$ is $\ell$-periodic, that is, $\sigma(x)=\left(j_{0}, \ldots, j_{\ell-1}, j_{0}, \ldots, j_{\ell-1}, \ldots\right)$, so we can describe it by a finite word $w=j_{0} \cdots j_{\ell-1}$ over the alphabet $\{1, \ldots, n\}$, which we will refer to as the word corresponding to $x$. This word can be read from the graph by recording the colors of the edges on the cycle, starting from $x$ and following the arrows backwards. Words corresponding to periodic points on the same cycle are conjugates, i.e., they can be obtained from each other by cyclic shifts. (For example, the words corresponding to $-1,-2,-6$ and -18 on Figure 1 are $3112,2311,1231$ and 1123, respectively.) Since our branching function systems have finitely many connected components, each containing a cycle (see Fact 2.4), their structure can be completely described by the words corresponding to periodic points (or cycles). Therefore, exploring periodic points and the corresponding words is essential in the study of representations of $\mathcal{P}_{n}$ and $\mathcal{O}_{n}$.

If a word $w$ is not the repetition of a shorter word, i.e., it cannot be written in the form $w=u \cdots u=u^{k}(k \geq 2)$, then $w$ is said to be a primitive word. Observe that every word is the power of a unique primitve word. The next corollary of Fact 2.5 states that the word corresponding to a periodic point is always primitive, and we also get a characterization of those primitive words that correspond to a periodic point. (Note that primitivity of the word corresponding to $x$ means that the shortest period of $\sigma(x)$ is the same as he shortest period of the sequence $x, R(x), R^{2}(x), \ldots$ In an arbitrary branching function system it is possible that the former is a proper divisor of the latter; however, this cannot happen for the special branching function systems considered here.)

Corollary 2.6. A word $w=j_{0} \cdots j_{\ell-1}$ over $\{1, \ldots, n\}$ corresponds to some periodic point if and only if $w$ is primitive and

$$
x=-\frac{d_{j_{\ell-1}} n^{\ell-1}+\cdots+d_{j_{1}} n+d_{j_{0}}}{n^{\ell}-1}
$$

is an integer. If this holds, then the integer $x$ defined by 2.2 is a periodic point on a cycle of length $\ell$, and $w$ is the word corresponding to $x$.

Proof. First we prove the second statement: let us assume that $w=j_{0} \cdots j_{\ell-1}$ is a primitive word such that the number given by $(2.2)$ is an integer. Then $x=d_{j_{0}}+d_{j_{1}} n+$ $\cdots+d_{j_{\ell-1}} n^{\ell-1}+n^{\ell} x$, which implies that $R(x)=\frac{x-d_{j_{0}}}{n}=d_{j_{1}}+\cdots+d_{j_{\ell-1}} n^{\ell-2}+n^{\ell-1} x$, since $x \equiv d_{j_{0}}(\bmod n)$, and $d_{j_{0}}$ is the only element of $D$ with this property. Continuing this way we get $R^{2}(x)=\frac{R(x)-d_{j_{1}}}{n}=d_{j_{2}}+\cdots+d_{j_{\ell-1}} n^{\ell-3}+n^{\ell-2} x$, etc., and after $\ell$ steps we obtain $R^{\ell}(x)=x$. This means that $x$ is indeed a periodic point; furthermore, 
if $u$ is the word corresponding to $x$ then $w=u^{k}$ with $\ell=k m$, where $m$ denotes the length of $u$ (that is, the length of the cycle that contains $x$ ). Since $w$ is a primitive word, we must have $k=1$, thus the length of the cycle containing $x$ is $\ell$ and the word corresponding to $x$ is $w$.

The argument above proves not only the second statement of the corollary, but also the "if" part of the first statement, thus it remains to prove the "only if" part. Let $w=j_{0} \cdots j_{\ell-1}$ be the word corresponding to some periodic point $y$. We can apply Fact 2.5 with $t=0$ to obtain that the right hand side of the equality (2.2) equals $y$, therefore it is an integer. Finally, we prove primitivity of $w$ : let $w=u^{k}$, where $u=j_{0} \cdots j_{m}$ is a primitive word and $\ell=k m$. Then we have $j_{i}=j_{i \bmod m}$ for every $i \in\{0, \ldots, \ell-1\}$, and this allows us to rewrite the right hand side of $(2.2)$ as follows:

$$
\begin{aligned}
x & =-\frac{\left(d_{j_{m-1}} n^{m-1}+\cdots+d_{j_{1}} n+d_{j_{0}}\right) \cdot\left(1+n^{m}+\cdots+n^{(k-1) m}\right)}{n^{\ell}-1} \\
& =-\frac{\left(d_{j_{m-1}} n^{m-1}+\cdots+d_{j_{1}} n+d_{j_{0}}\right) \cdot \frac{n^{k m}-1}{n^{m}-1}}{n^{\ell}-1}=-\frac{d_{j_{m-1}} n^{m-1}+\cdots+d_{j_{1}} n+d_{j_{0}}}{n^{m}-1} .
\end{aligned}
$$

Applying the first paragraph of this proof to the primitive word $u$, we see that $x$ belongs to a cycle of length $m$ (and the word corresponding to $x$ is $u$ ). Consequently, $k=1$ and $u=w$, proving that $w$ is indeed primitive.

Remark 2.7. The proof of Corollary 2.6 shows that if the number $x$ given by $(2.2)$ is an integer, then it is a periodic point even if the word $w$ is not primitive. Moreover, if $w=u^{k}$, where $u$ is a primitive word, then the word corresponding to $x$ is $u$. Thus, the periodic points are exactly the integers of the form

$$
\frac{a_{\ell-1} n^{\ell-1}+\cdots+a_{1} n+a_{0}}{1-n^{\ell}} \quad\left(\ell \in \mathbb{N}, a_{0}, \ldots, a_{\ell-1} \in D\right) .
$$

Two such expressions yield the same number if and only if the words describing the coefficients $a_{0}, \ldots, a_{\ell-1}$ are the powers of the same primitive word. In particular, different expressions of the same length $\ell$ give different periodic points.

Fact 2.8. The words corresponding to periodic points on different cycles are not conjugate.

Proof. Let $v$ and $w$ be words corresponding to periodic points $x$ and $y$ that lie on different cycles, and suppose that $v$ and $w$ are conjugates. Then there exists a point $x^{\prime}$ on the same cycle as $x$ such that the word corresponding to $x^{\prime}$ is $w$. However, according to Corollary 2.6, a periodic point is uniquely determined by the corresponding word by 2.2 . This implies that $x^{\prime}=y$, hence $x$ and $y$ belong to the same cycle, contrary to our assumption.

Summarizing the above facts, we can say that the structure of the branching function system corresponding to $d_{1}, \ldots, d_{n}$ is determined by a finite set of primitive words over the alphabet $\{1, \ldots, n\}$, each considered up to conjugacy. For the sake of canonicity, it is customary to choose the lexicographically least word from each conjugacy class; these words are called Lyndon words. (The Lyndon words describing the three cycles of Figure 1 are 1123, 23 and 1.) One possible approach to find these Lyndon words is to follow the trajectories of the elements of $\mathcal{A}(D)$. Another possibility is to determine all numbers of the form $a_{\ell-1} n^{\ell-1}+\cdots+a_{1} n+a_{0}$ with $a_{0}, \ldots, a_{\ell-1} \in D$ that are divisible by $n^{\ell}-1$ (cf. Remark 2.7). Since $\mathcal{A}(D)$ is finite, both searches can be completed in a finite number of steps (note that we must have $\ell \leq\left|B_{\infty}\right| \leq|\mathcal{A}|$ ).

Corollary 2.9. It is decidable whether two one-dimensional strong affine representations of $\mathcal{P}_{n}$ are equivalent.

Remark 2.10. Let $\sigma(x)=\left(j_{0}, j_{1}, \ldots\right)$ and let us write out the representations of $x$ considered in the proof of Fact 2.5 for $k=1,2,3, \ldots$ :

$$
x=d_{j_{0}}+n R(x)=d_{j_{0}}+n d_{j_{1}}+n^{2} R^{2}(x)=d_{j_{0}}+n d_{j_{1}}+n^{2} d_{j_{2}}+n^{3} R^{3}(x)=\cdots .
$$


It would be natural to extend this to an infinite expansion of $x$ :

$$
x=d_{j_{0}}+n d_{j_{1}}+n^{2} d_{j_{2}}+n^{3} d_{j_{3}}+\cdots .
$$

Of course, this infinite series does not converge in general. However, we can infer from 2.3 that $x \equiv d_{j_{0}}(\bmod n), x \equiv d_{j_{0}}+n d_{j_{1}}\left(\bmod n^{2}\right), x \equiv d_{j_{0}}+n d_{j_{1}}+n^{2} d_{j_{2}}\left(\bmod n^{3}\right)$, etc. Moreover, since the expansion is periodic, we can sum the right hand side of (2.3) formally by letting $1+n^{\ell}+n^{2 \ell}+\cdots=\frac{1}{1-n^{\ell}}$. Although this geometric series is clearly divergent, this formal evaluation gives actually the correct value of $x$. Indeed, let $a=d_{j_{0}}+n d_{j_{1}}+\cdots+n^{t-1} d_{j_{t-1}}$ and $b=d_{j_{t}} n^{t}+\cdots+d_{j_{t+\ell-1}} n^{t+\ell-1}$ (with $t$ and $\ell$ being the same as in Fact 2.5); then we can rewrite 2.3 as $x=a+b+b n^{\ell}+b n^{2 \ell}+\cdots=a+\frac{b}{1-n^{\ell}}$, and this is the same as 2.1.

One can regard 2.3) as a representation of $x$ in a number system with radix $n$ and digits $d_{1}, \ldots, d_{n}$. As mentioned in Subection $1.5, B_{\infty}(0, \ldots, n-1)=\{-1,0\}$ (see also Theorem 3.1. This means that using the standard digits $0, \ldots, n-1$, the sequence of digits in the expansion of every integer is eventually constant 0 or constant $n-1$, namely for nonnegative integers the digits are eventually 0 (as it is well known), and for negative integers the digits are eventually $n-1$. For instance, the representation of $x=-1$ is $-1=(n-1)+(n-1) n+(n-1) n^{2}+\cdots$, which can be verfied using the formal summation $1+n+n^{2}+\cdots=\frac{1}{1-n}$.

As another example, we can read from Figure 1 that 50 can be represented in the number system with radix 3 and digits $0,1,53$ as

$$
\begin{aligned}
50 & =d_{3}+n d_{3}+n^{2} d_{1}+n^{3} d_{1}+n^{4} d_{2}+n^{5} d_{3}+n^{6} d_{1}+n^{7} d_{1}+n^{8} d_{2}+\cdots \\
& =53+53 \cdot 3+0 \cdot 3^{2}+0 \cdot 3^{3}+1 \cdot 3^{4}+53 \cdot 3^{5}+0 \cdot 3^{6}+0 \cdot 3^{7}+1 \cdot 3^{8}+\cdots \\
& =53+240 \cdot\left(1+3^{4}+3^{8}+\cdots\right) .
\end{aligned}
$$

Evaluating $1+3^{4}+3^{8}+\cdots$ formally as $\frac{1}{1-3^{4}}$, we indeed get $53+240 \cdot \frac{1}{-80}=53-3=50$.

Finally, we state an elementary identity about integer parts that will be needed later; its proof is left to the reader.

Lemma 2.11. For all $x \in \mathbb{R}$ and $n \in \mathbb{N}$ we have $\sum_{k=0}^{n-1}\left\lfloor x+\frac{k}{n}\right\rfloor=\lfloor n x\rfloor$.

\section{Arithmetic SEquences}

In this section we investigate periodic points and cycles in the case when the parameters form an arithmetic sequence. For notational convenience, we shift the indices by 1 (as it is done in Section 5 of [16]): instead of $d_{1}, \ldots, d_{n}$ we shall work with $d_{0}, \ldots, d_{n-1}$, and we will use words over $\{0, \ldots, n-1\}$ to describe the cycles. Thus, we assume throughout this section that $d_{0}, \ldots, d_{n-1}$ is an arithmetic sequence: $d_{i}=d_{0}+i h$ for $i=0, \ldots, n-1$, where $h \in \mathbb{N}$ is relatively prime to $n$ and $0 \leq d_{0}<n-1$. Observe that $\mathcal{A}=\{-h, \ldots,-1,0\}$ if $d_{0}=0$ and $\mathcal{A}=\{-h, \ldots,-1\}$ if $0<d_{0}<n-1$.

Theorem 3.1. If $d_{0}<\cdots<d_{n-1}$ is an arithmetic sequence, then $B_{\infty}=\mathcal{A}$, therefore the number of periodic points is $h+1$ or $h$ (depending on whether $d_{0}=0$ or not).

Proof. Since $\mathcal{A}$ is a positively invariant set (Lemma 2.3) and $B_{\infty} \subseteq \mathcal{A}$ (Fact 2.4), it suffices to prove that the restriction of $R$ to $\mathcal{A}$ is bijective. In fact, it is sufficient to establish injectivity, as $\mathcal{A}$ is finite. Let $x, y, z \in \mathcal{A}$ such that $R(x)=R(y)=z$ and $x<y$. Then we have $x=n z+d_{i}, y=n z+d_{j}$ for some $i, j \in\{1, \ldots, n\}$, therefore $y-x=d_{j}-d_{i}=h(j-i) \geq h$. If $d_{0} \neq 0$ then this is already a contradiction, as the diameter of the set $\mathcal{A}$ is $h-1$ in this case. If $d_{0}=0$, then the diameter of $\mathcal{A}$ is $h$, and this implies that $x=\min \mathcal{A}=-h$ and $y=\max \mathcal{A}=0$. However, $R(-h)=-h$ and $R(0)=0$, contradicting the assumption $R(x)=R(y)$.

The next lemma is essentially just a reformulation of Corollary 2.6 for arithmetic sequences. 
Lemma 3.2. Assume that the sequence $d_{0}<\cdots<d_{n-1}$ is arithmetic, let $w=$ $j_{0} \cdots j_{\ell-1}$ be a primitive word over $\{0, \ldots, n-1\}$ and let $\overleftarrow{w} \in\left\{0,1, \ldots, n^{\ell}-1\right\}$ be the nonnegative integer given by the base-n representation determined by the reverse of $w$ :

$$
\overleftarrow{w}=\overline{j_{\ell-1} \cdots j_{1} j_{0}} n=\sum_{i=0}^{\ell-1} j_{i} n^{i}
$$

Then $w$ corresponds to a cycle if and only if

$$
n^{\ell}-1 \mid \frac{d_{0}\left(n^{\ell}-1\right)}{n-1}+h \overleftarrow{w}
$$

Proof. Substituting $d_{0}+j_{i} h$ for $d_{j_{i}}$ in the numerator of the right hand side of $(2.2)$, we obtain

$$
\sum_{i=0}^{\ell-1} d_{0} n^{i}+\sum_{i=0}^{\ell-1} j_{i} h n^{i}=\frac{d_{0}\left(n^{\ell}-1\right)}{n-1}+h \overleftarrow{w}
$$

By Corollary 2.6, $w$ corresponds to a periodic point if and only if the above number is divisible by $n^{\ell}-1$.

Now we are ready to generalize Theorem 5.5 of [16] by relating the words corresponding to periodic points to base- $n$ expansions of certain fractions. (Note that for $n=2$ we must have $d_{0}=0$, hence the following theorem indeed contains Theorem 5.5 of [16] as a special case.)

Theorem 3.3. Assume that $d_{0}<\cdots<d_{n-1}$ is an arithmetic sequence. If $d_{0}=0$ then the words corresponding to the periodic points are the reverses of the periods of the base-n representations of the fractions $\frac{0}{h}, \ldots, \frac{h}{h}$. If $0<d_{0}<n-1$, then the corresponding words are the reverses of the periods of the base-n representations of $\frac{1}{h}-\frac{d_{0}}{h(n-1)}, \ldots, \frac{h}{h}-\frac{d_{0}}{h(n-1)}$.

Proof. Let $x \in B_{\infty}$ and let $w=j_{0} \cdots j_{\ell-1}$ be a primitive word over $\{0, \ldots, n-1\}$. By (the proof of) Lemma $3.2, w$ corresponds to $x$ if and only if $-x=\frac{d_{0}}{n-1}+\frac{h \overleftarrow{w}}{n^{\ell}-1}$, which is equivalent to

$$
\begin{aligned}
-\frac{x+\frac{d_{0}}{n-1}}{h} & =\frac{\overleftarrow{w} / n^{\ell}}{1-1 / n^{\ell}}=\overleftarrow{w}\left(\frac{1}{n^{\ell}}+\frac{1}{n^{2 \ell}}+\cdots\right) \\
& =j_{\ell-1} \frac{1}{n}+\cdots+j_{0} \frac{1}{n^{\ell}}+j_{\ell-1} \frac{1}{n^{\ell+1}}+\cdots+j_{0} \frac{1}{n^{2 \ell}}+\cdots \\
& =0 .{\frac{j}{\ell-1} \cdots j_{0} j_{\ell-1} \cdots j_{0} \cdots}_{n} .
\end{aligned}
$$

Thus, the word corresponding to $x \in B_{\infty}$ is the reverse of the period in the base- $n$ expansion of $-\frac{x}{h}-\frac{d_{0}}{h(n-1)}$. Taking into account that $B_{\infty}=\{-h, \ldots,-1,0\}$ if $d_{0}=0$ and $B_{\infty}=\{-h, \ldots,-1\}$ if $0<d_{0}<n-1$, we obtain the (negatives of the) fractions listed in the statement of the theorem.

Corollary 3.4. If $D$ is an arithmetic sequence, then the length of the cycle containig the periodic point

$$
x \in B_{\infty}(D)
$$

equals the multiplicative order of $n$ modulo $\frac{h(n-1)}{\operatorname{gcd}\left(x(n-1)+d_{0}, h(n-1)\right)}$.

Proof. It is well known that if $a, b \in \mathbb{N}$ are relatively prime and $b$ is also relatively prime to $n$, then the length of the period of the base- $n$ representation of $\frac{a}{b}$ is the order of $n$ modulo $b$. We have seen in the proof of Theorem 3.3 that the length of the cycle containing $x \in B_{\infty}$ is the length of the period of the base- $n$ expansion of $\frac{x(n-1)+d_{0}}{h(n-1)}$; therefore, it only remains to observe that after simplification the denominator becomes $\frac{h(n-1)}{\operatorname{gcd}\left(x(n-1)+d_{0}, h(n-1)\right)}$, which is clearly relatively prime to $n$, as both $h$ and $n-1$ are. 
Corollary 3.5. For any finite set $C$ of primitive words over the alphabet $\{0, \ldots, n-$ $1\}$ there exists an arithmetic sequence $d_{0}<\cdots<d_{n-1}$ such that the set of words corresponding to the elements of $B_{\infty}\left(d_{0}, \ldots, d_{n-1}\right)$ contains $C$.

Proof. By Lemma 3.2 , it suffices to find $d_{0}$ and $h$ such that

$$
\frac{d_{0}\left(n^{|w|}-1\right)}{n-1}+h \overleftarrow{w} \equiv 0\left(\bmod n^{|w|}-1\right)
$$

for every $w \in C$, where $|w|$ denotes the length of $w$. Clearly, for $d_{0}=0$ and $h=$ $\operatorname{lcm}\left\{n^{|w|}-1: w \in C\right\}$ each of these congruences are satisfied (note that $h$ is relatively prime to $n$ ).

We finish this section by showing that the only equivalences between representations (or branching function systems) given by arithmetic sequences are the trivial ones of Fact 2.2

Theorem 3.6. Let $0 \leq d_{0}, d_{0}^{\prime}<n-1$ and let $h, h^{\prime}$ be relatively prime to $n$. The representations of $\mathcal{P}_{n}$ arising from the arithmetic sequences $d_{0}, d_{0}+h, \ldots, d_{0}+(n-1) h$ and $d_{0}^{\prime}, d_{0}^{\prime}+h^{\prime}, \ldots, d_{0}^{\prime}+(n-1) h^{\prime}$ are equivalent if and only if $d_{0}=d_{0}^{\prime}$ and $h=h^{\prime}$.

Proof. Let the two representations given in the statement of the theorem be equivalent, and first let us assume that $h \neq h^{\prime}$; without loss of generality we can suppose that $h<h^{\prime}$. Since equivalent representations have the same number of periodic points, Theorem 3.1 implies that $d_{0}=0, d_{0}^{\prime} \neq 0$ and $h^{\prime}=h+1$. Then $0 \in B_{\infty}\left(d_{0}, \ldots, d_{n-1}\right)$ and the corresponding word is 0 (of length one). However, since $n-1 \nmid d_{0}^{\prime}$, this word corresponds to no element of $B_{\infty}\left(d_{0}^{\prime}, \ldots, d_{n-1}^{\prime}\right)$, contradicting the equivalence of the representations.

Now let us assume that $h=h^{\prime}$ but $d_{0} \neq d_{0}^{\prime}$. Recall that two representations are equivalent if and only if the sets of words determined by their periodic points are the same. Let $w$ be a word of length $\ell$ corresponding to some element of $B_{\infty}\left(d_{0}, \ldots, d_{n-1}\right)$; then $w$ also corresponds to some element of $B_{\infty}\left(d_{0}^{\prime}, \ldots, d_{n-1}^{\prime}\right)$. Lemma 3.2 implies that

$$
\frac{d_{0}\left(n^{\ell}-1\right)}{n-1}+h \overleftarrow{w} \equiv \frac{d_{0}^{\prime}\left(n^{\ell}-1\right)}{n-1}+h^{\prime} \overleftarrow{w} \equiv 0\left(\bmod n^{\ell}-1\right)
$$

which in turn implies that $d_{0} \equiv d_{0}^{\prime}(\bmod n-1)$, as $h=h^{\prime}$. However, this is impossible, since $0 \leq d_{0}, d_{0}^{\prime}<n-1$ and $d_{0} \neq d_{0}^{\prime}$.

\section{MANY PERIODIC POINTS}

Arithmetic sequences give rise to branching function systems where the set of periodic points is "as large as possible", i.e., $B_{\infty}=\mathcal{A}$. In this section we characterize sequences $d_{1}, \ldots, d_{n}$ that have the same property; as we shall see, these are "almost arithmetic sequences".

Theorem 4.1. Let $d_{1}<\cdots<d_{n}$, let $\mathcal{I}$ denote the interval $\left[-\frac{d_{n}}{n-1},-\frac{d_{1}}{n-1}\right]$ as before, and let $\mathcal{I}_{i}=\frac{1}{n} \mathcal{I}-\frac{1}{n} d_{i}$ for $i=1, \ldots, n$. The following conditions are equivalent:

(i) $B_{\infty}=\mathcal{A}$;

(ii) $\mathcal{A} \subseteq \mathcal{I}_{1} \cup \cdots \cup \mathcal{I}_{n}$;

(iii) for all $i \in\{1, \ldots, n-1\}$ we have

$$
\left\lfloor\frac{d_{1}}{n(n-1)}+\frac{d_{i+1}}{n}\right\rfloor=\left\lfloor\frac{d_{n}}{n(n-1)}+\frac{d_{i}}{n}\right\rfloor .
$$

Proof. Just as in the proof of Theorem 3.1, we can see that $B_{\infty}=\mathcal{A}$ if and only if the restriction of $R$ to $\mathcal{A}$ is bijective. Since $\mathcal{A}$ is finite, bijectivity is equivalent to surjectivity in this case, thus (i) holds if and only if $R^{-1}(x) \cap \mathcal{A}=R^{-1}(x) \cap \mathcal{I} \neq \emptyset$ for all $x \in \mathcal{A}$. This latter condition means that for every $x \in \mathcal{A}$ there exists an $i \in\{1, \ldots, n\}$ such that $n x+d_{i} \in \mathcal{I}$, i.e., $x \in \mathcal{I}_{i}$. This proves the equivalence of (i) and (ii)

The intervals $\mathcal{I}_{i}$ are all translates of the interval $\frac{1}{n} \mathcal{I}$. The leftmost one of these intervals is $\mathcal{I}_{n}$, and the left endpoint of $\mathcal{I}_{n}$ coincides with the left endpoint of $\mathcal{I}$; 
similarly, the right endpoint of $\mathcal{I}_{1}$ coincides with the right endpoint of $\mathcal{I}$. Therefore, condition (ii) holds if and only if there is no integer between the right endpoint of $\mathcal{I}_{i+1}$ and the left endpoint of $\mathcal{I}_{i}$ for every $i \in\{1, \ldots, n-1\}$ :

$$
\nexists x \in \mathbb{Z}:-\frac{d_{1}}{n(n-1)}-\frac{d_{i+1}}{n}<x<-\frac{d_{n}}{n(n-1)}-\frac{d_{i}}{n} .
$$

Since $1 \neq i+1$, we have $d_{1} \not \equiv d_{i+1}(\bmod n)$, and this implies that $-\frac{d_{1}}{n(n-1)}-\frac{d_{i+1}}{n}$ is not an integer; similarly, the right hand side of (4.1) is not an integer either, as $n \neq i$. Hence 4.1 is equivalent to $\left\lfloor-\frac{d_{1}}{n(n-1)}-\frac{d_{i+1}}{n}\right\rfloor \geq\left\lfloor-\frac{d_{n}}{n(n-1)}-\frac{d_{i}}{n}\right\rfloor$, which, in turn, is equivalent to

$$
\left\lfloor\frac{d_{1}}{n(n-1)}+\frac{d_{i+1}}{n}\right\rfloor \leq\left\lfloor\frac{d_{n}}{n(n-1)}+\frac{d_{i}}{n}\right\rfloor .
$$

This shows that condition (ii) is satisfied if and only if the inequality 4.2 is true for $i=1, \ldots, n-1$. In order to prove the equivalence of (ii) and (iii) we just need to verify that if 4.2 holds for all $i \in\{1, \ldots, n-1\}$, then we actually have equality in 4.2 for every $i$. This will follow immediately from the observation that adding the inequalities 4.2 for $i=1, \ldots, n-1$, we obtain the same values on the left hand side and on the right hand side. Indeed, summing the left hand sides of 4.2 for $i=1, \ldots, n-1$ we get

$$
\begin{aligned}
\sum_{i=1}^{n-1}\left\lfloor\frac{d_{1}}{n(n-1)}+\frac{d_{i+1}}{n}\right\rfloor & =\sum_{j=1}^{n}\left\lfloor\frac{d_{1}}{n(n-1)}+\frac{n q_{j}+r_{j}}{n}\right\rfloor-\left\lfloor\frac{d_{1}}{n(n-1)}+\frac{d_{1}}{n}\right\rfloor \\
& \stackrel{(\mathrm{a})}{=} \sum_{j=1}^{n} q_{j}+\sum_{k=0}^{n-1}\left\lfloor\frac{d_{1}}{n(n-1)}+\frac{k}{n}\right\rfloor-\left\lfloor\frac{d_{1}}{n-1}\right\rfloor \\
& \stackrel{(\mathrm{b})}{=} \sum_{j=1}^{n} q_{j},
\end{aligned}
$$

where in step (a) we used the fact that $\left\{r_{1}, \ldots, r_{n}\right\}=\{0, \ldots, n-1\}$, as $d_{1}, \ldots, d_{n}$ is a complete system of residues modulo $n$, and in step (b) we applied Lemma 2.11 with $x=\frac{d_{1}}{n(n-1)}$. A similar calculation shows that the sum of the right hand sides of $\sqrt{4.2}$ for $i=1, \ldots, n-1$ is also $\sum_{j=1}^{n} q_{j}$.

Remark 4.2. Assuming (without loss of generality) that $0 \leq d_{1}<n-1$, condition (iii) of Theorem 4.1 yields

$$
q_{i+1}-q_{i}=\left\lfloor\frac{d_{n}}{n(n-1)}+\frac{r_{i}}{n}\right\rfloor \in\left\{\left\lfloor\frac{d_{n}}{n(n-1)}\right\rfloor,\left\lfloor\frac{d_{n}}{n(n-1)}\right\rfloor+1\right\} .
$$

Thus in this case $q_{1}, \ldots, q_{n}$ is almost an arithmetic sequence: the difference of consecutive entries can assume at most two different values (which are consecutive integers), and then the numbers $d_{i}=n q_{i}+r_{i}$ are also quite evenly distributed in the interval $\left[d_{1}, d_{n}\right]$. So we can say informally that $B_{\infty}=\mathcal{A}$ if and only if $d_{1}, \ldots, d_{n}$ is not far from being an arithmetic sequence. (Note that this intuitive interpretation of Theorem 4.1 can be misleading; see Example 4.3.) It is straightforward to verify that $d_{1}, \ldots, d_{n}$ form an arithmetic sequence if and only if the equality in condition (iii) holds even without taking integer parts.

From (4.3) we also obtain the following explicit formula for $q_{i}$ (taking into account that $\left.q_{1}=0\right)$ :

$$
q_{i}=\left\lfloor\frac{d_{n}}{n(n-1)}+\frac{r_{1}}{n}\right\rfloor+\cdots+\left\lfloor\frac{d_{n}}{n(n-1)}+\frac{r_{i-1}}{n}\right\rfloor .
$$

This means that if we prescribe the residues $r_{1}, \ldots, r_{n}$ and we also fix $d_{1}$ and $d_{n}$, then there is at most one possibility for the numbers $d_{2}, \ldots, d_{n-1}$ such that $B_{\infty}(D)=$ $\mathcal{A}(D)$. However the numbers $d_{2}, \ldots, d_{n-1}$ calculated by 4.4 do not necessarily form 
an increasing sequence (cf. Example 4.4). In this case we can conclude from Theorem 4.1 that there is no branching function system with the given values for the residues and for $d_{1}$ and $d_{n}$ with $B_{\infty}=\mathcal{A}$.

Example 4.3. The following table gives the data for a branching function system with $n=10$ and $B_{\infty}=\mathcal{A}$. We can observe that $q_{i+1}-q_{i} \in\{1,2\}$ for $i=1, \ldots, 9$, in accordance with (4.3). The last line of the table gives the members of the arithmetic sequence $s_{i}$ with $s_{1}=d_{1}=0$ and $s_{10}=d_{10}=141$, rounded to the nearest integer, i.e., $s_{i}=\left\lfloor(i-1) \cdot \frac{141}{9}\right\rceil$. Theorem 4.1 and Remark 4.2 might give the impression that $d_{i}$ should be one of the integers that are closest to $s_{i}$ and congruent to $r_{i}$ modulo $n$. However, this is not true; for instance, one would expect that $d_{5}$ is either 56 or 66 , but the actual value is $d_{5}=76$. The case $i=7$ is even more counterintuitive: $s_{7}=94$ is already congruent to $r_{7}=4$ modulo 10 (and we do not even need to round when computing $s_{7}$, as $6 \cdot \frac{141}{9}$ is an integer), yet $d_{7}=114$ is quite far from $s_{7}$.

\begin{tabular}{|c||r|r|r|r|r|r|r|r|r|r|}
$i$ & 1 & 2 & 3 & 4 & 5 & 6 & 7 & 8 & 9 & 10 \\
\hline \hline$r_{i}$ & 0 & 9 & 8 & 7 & 6 & 5 & 4 & 3 & 2 & 1 \\
$q_{i}$ & 0 & 1 & 3 & 5 & 7 & 9 & 11 & 12 & 13 & 14 \\
$d_{i}$ & 0 & 19 & 38 & 57 & 76 & 95 & 114 & 123 & 132 & 141 \\
$s_{i}$ & 0 & 16 & 31 & 47 & 63 & 78 & 94 & 110 & 125 & 141
\end{tabular}

Example 4.4. Let us try to find a branching function system with $B_{\infty}=\mathcal{A}$ for $n=4$ such that it satisfies $r_{1}=2, r_{2}=3, r_{3}=1, r_{4}=0$ and $d_{1}=2, d_{4}=8$. The values computed from (4.4) are given in the table below. We can see that $d_{3}>d_{4}$, and this means that no branching function system with the given parameters satisfies $B_{\infty}=\mathcal{A}$. (Note that if we switch $r_{2}$ and $r_{3}$ then we have $B_{\infty}=\mathcal{A}$ with $d_{2}=5, d_{3}=7$.)

\begin{tabular}{|c||c|c|c|c|}
$i$ & 1 & 2 & 3 & 4 \\
\hline \hline$r_{i}$ & 2 & 3 & 1 & 0 \\
$q_{i}$ & 0 & 1 & 2 & 2 \\
$d_{i}$ & 2 & 7 & 9 & 8 \\
& & & &
\end{tabular}

\section{A SINGLE PERIODIC POINT}

In 4, the authors raised the question when a representation of $\mathcal{O}_{n}$ has a single periodic point (these representations are necessarily irreducible). Jeong has given some examples in [15] of such representations. In this section we provide an infinite family of representations having a single periodic point and give some sporadic examples indicating that there are far more such representations.

If $d_{1}, \ldots, d_{n}$ are consecutive integers, then the number of periodic points is either one or two, by Theorem 3.1. In the next theorem we investigate how the number of periodic points changes when one of the $d_{i}$ 's is replaced by $d_{i}+n^{k}$. We will see that for most choices of $d_{i}$ the number of periodic points does not change, but in certain special cases the number of periodic points increases exponentially with $k$. By the remark following Fact 2.2, we may assume without loss of generality that $\left(d_{1}, \ldots, d_{n}\right)=(b, \ldots, b+n-1)$ with $0 \leq b \leq n-2$.

Theorem 5.1. Let $0 \leq b \leq n-2$, let $r \in\{b, b+1, \ldots, b+n-1\}$ and $k \in \mathbb{N}$. The number of periodic points corresponding to

$$
\left(d_{1}, \ldots, d_{n}\right)=\left(b, \ldots, r-1, r+n^{k}, r+1, \ldots, b+n-1\right)
$$

is given by Table 1 .

Proof. Let $\left(d_{1}, \ldots, d_{n}\right)=\left(b, \ldots, r-1, r+n^{k}, r+1, \ldots, b+n-1\right)$, i.e., $d_{i}=b+i-1$ for $i \neq r-b+1$ and $d_{i}=b+i-1+n^{k}$ for $i=r-b+1$. Let $x$ be an arbitrary periodic point on a cycle of length $\ell$, let $\sigma(x)=\left(j_{0}, j_{1}, \ldots\right)$, and let $a_{i}=d_{j_{i}}$. By Corollary 2.6. 


\begin{tabular}{cl||l} 
& & $\left|B_{\infty}\right|$ \\
\hline \hline$b=0$ & $r=0$ & 1 \\
$b=0$ & $r \in\{1, \ldots, n-3\}$ & 2 \\
$b=0$ & $r \in\{n-2, n-1\}$ & $2^{k}+1$ \\
\hline $1 \leq b \leq n-3$ & $r \in\{n-2, n-1\}$ & $2^{k}$ \\
$1 \leq b \leq n-3$ & $r \notin\{n-2, n-1\}$ & 1 \\
\hline$b=n-2$ & $r \in\{n-2, n-1\}$ & $2^{k}$ \\
$b=n-2$ & $r \in\{n, \ldots, 2 n-4\}$ & 1 \\
$b=n-2$ & $r=2 n-3$ & 2
\end{tabular}

TABLE 1. The number of periodic points for some special branching function systems (see Theorem 5.1)

we have $x=-y /\left(n^{\ell}-1\right)$, where $y=n^{\ell-1} a_{\ell-1}+\cdots+n a_{1}+a_{0}$; moreover, $\left\{a_{i}\right\}$ is a periodic sequence whose shortest period is $\ell$.

Let us define the following two modified sequences of coefficients (here, and in the sequel, $\ominus$ and $\oplus$ stand for subtraction and addition modulo $\ell$, respectively):

$$
a_{i}^{\prime}:=\left\{\begin{array}{rl}
a_{i}-n^{k}, & \text { if } a_{i}=r+n^{k} ; \\
a_{i}, & \text { otherwise; }
\end{array} \quad a_{i}^{\prime \prime}:=\left\{\begin{aligned}
a_{i}^{\prime}+1, & \text { if } a_{i \ominus k}^{\prime}=r \\
a_{i}^{\prime}, & \text { otherwise }
\end{aligned}\right.\right.
$$

Note that $0 \leq a_{i}^{\prime \prime} \leq b+n \leq 2 n-2$ for all $i \in\{0, \ldots, \ell-1\}$. We can now express $y$ modulo $n^{\ell}-1$ with these new coefficients, using $s \equiv t(\bmod \ell) \Longrightarrow n^{s} \equiv n^{t}\left(\bmod n^{\ell}-1\right)$ :

$$
\begin{aligned}
y & =\sum_{i=0, \ldots, \ell-1} a_{i}^{\prime} n^{i}+\sum_{\substack{i=0, \ldots, \ell-1 \\
a_{i}=r+n^{k}}} n^{k} n^{i} \equiv \sum_{i=0, \ldots, \ell-1} a_{i}^{\prime} n^{i}+\sum_{\substack{i=0, \ldots, \ell-1 \\
a_{i}=r+n^{k}}} n^{i \oplus k} \\
& =\sum_{j=0, \ldots, \ell-1} a_{j}^{\prime} n^{j}+\sum_{\substack{j=0, \ldots, \ell-1 \\
a_{j \ominus k}^{\prime}=r}} n^{j}=\sum_{j=0, \ldots, \ell-1} a_{j}^{\prime \prime} n^{j}=: y^{\prime \prime}\left(\bmod n^{\ell}-1\right) .
\end{aligned}
$$

Since $0 \leq a_{i}^{\prime \prime} \leq 2 n-2$, we have $0 \leq y^{\prime \prime} \leq(2 n-2) \sum_{j=0}^{\ell-1} n^{j}=2\left(n^{\ell}-1\right)$. On the other hand, $y^{\prime \prime} \equiv y \equiv 0\left(\bmod n^{\ell}-1\right)$, therefore $y^{\prime \prime} \in\left\{0, n^{\ell}-1,2\left(n^{\ell}-1\right)\right\}$. We examine these three cases separately.

1) If $y^{\prime \prime}=0$, then $a_{i}^{\prime \prime}=0$ for all $i$, hence $a_{i}^{\prime}=0$ and $a_{i \ominus k}^{\prime} \neq r$ for all $i$. This happens if and only if $b=0, r \neq 0$ and $a_{i}=0$ for all $i$. This gives us the periodic point $x=0$ with $\ell=1$.

2) If $y^{\prime \prime}=n^{\ell}-1$, then $a_{i}^{\prime \prime}=n-1$ for all $i$. Indeed, $-1 \equiv n^{\ell}-1=y^{\prime \prime} \equiv a_{0}^{\prime \prime}(\bmod n)$ and $0 \leq a_{0}^{\prime \prime} \leq 2 n-2$ imply that $a_{0}^{\prime \prime}=n-1$. Then we have $-1 \equiv n^{\ell-1}-1=$ $\left(y^{\prime \prime}-\bar{a}_{0}^{\prime \prime}\right) / n \equiv a_{1}^{\prime \prime}(\bmod n)$ and $0 \leq a_{1}^{\prime \prime} \leq 2 n-2$, hence $a_{1}^{\prime \prime}=n-1$. Continuing this way one can prove by induction on $i$ that $a_{i}^{\prime \prime}=n-1$ for every $i$. This happens if and only if for each $i$ we have either $a_{i}^{\prime}=n-1, a_{i \ominus k}^{\prime} \neq r$ or $a_{i}^{\prime}=n-2, a_{i \ominus k}^{\prime}=r$. Here we can distinguish three subcases.

2a) If $r \notin\{n-2, n-1\}$, then $a_{i}^{\prime}=a_{i}=n-1$ for all $i$, therefore $\ell=1$ and we obtain the periodic point $x=-1$.

2b) If $r=n-2$, then $a_{i}^{\prime} \in\{n-1, n-2\}$ and $a_{i \ominus k}^{\prime}=a_{i}^{\prime}$ for all $i$. Thus $\left\{a_{i}\right\}$ is a $k$-periodic sequence with entries $n-1$ and $n-2$. (Recall that the shortest period of $\left\{a_{i}\right\}$ was $\ell$, hence $\ell \mid k$.) By Remark 2.7. we obtain $2^{k}$ different periodic points in this case.

2c) If $r=n-1$, then $a_{i}^{\prime} \in\{n-1, n-2\}$ and $a_{i \ominus k}^{\prime} \neq a_{i}^{\prime}$ for all $i$, which implies $a_{i \ominus 2 k}^{\prime}=a_{i}^{\prime}$. Thus $\left\{a_{i}\right\}$ is a $2 k$-periodic sequence with entries $n-1$ and $n-2$ such that the first half of the period uniquely determines the second half: $a_{i \oplus k}^{\prime}=2 n-3-a_{i}^{\prime}$. (Here we must have $\ell \mid 2 k$ and $\ell \nmid k$.) Just like in case $2 \mathrm{~b}$ ), we get $2^{k}$ periodic points. 


\begin{tabular}{|c|c|c|c|c|c|c|c|}
\hline & & 1) & $2 \mathrm{a})$ & 2b) & 2c) & 3) & $\left|B_{\infty}\right|$ \\
\hline$b=0$ & $r=0$ & & 1 & & & & 1 \\
\hline$b=0$ & $r \in\{1, \ldots, n-3\}$ & 1 & 1 & & & & 2 \\
\hline$b=0$ & $r=n-2$ & 1 & & $2^{k}$ & & & $2^{k}+1$ \\
\hline$b=0$ & $r=n-1$ & 1 & & & $2^{k}$ & & $2^{k}+1$ \\
\hline $1 \leq b \leq n-3$ & $r=n-2$ & & & $2^{k}$ & & & $2^{k}$ \\
\hline $1 \leq b \leq n-3$ & $r=n-1$ & & & & $2^{k}$ & & $2^{k}$ \\
\hline $1 \leq b \leq n-3$ & $r \notin\{n-2, n-1\}$ & & 1 & & & & 1 \\
\hline$b=n-2$ & $r=n-2$ & & & $2^{k}$ & & & $2^{k}$ \\
\hline$b=n-2$ & $r=n-1$ & & & & $2^{k}$ & & $2^{k}$ \\
\hline$b=n-2$ & $r \in\{n, \ldots, 2 n-4\}$ & & 1 & & & & 1 \\
\hline$b=n-2$ & $r=2 n-3$ & & 1 & & & 1 & 2 \\
\hline
\end{tabular}

TABLE 2. The number of periodic points in the different cases in the proof of Theorem 5.1

3) If $y^{\prime \prime}=2\left(n^{\ell}-1\right)$, then $a_{i}^{\prime \prime}=2 n-2$ for all $i$, hence $a_{i}^{\prime}=2 n-3$ and $a_{i \ominus k}^{\prime}=r$ for all $i$. This happens if and only if $b=n-2, r=2 n-3$ and $a_{i}=2 n-3+n^{k}$ for all $i$. Just as in the first case, this implies $\ell=1$, and the corresponding periodic point is $x=-\frac{2 n-3+n^{k}}{n-1}$.

Now only some bookkeeping is needed to finish the proof: one has to count the number of periodic points in the above cases, and determine which cases can occur for given values of $b$ and $r$. The results are given in Table 2

Remark 5.2. The previous theorem concentrated on just one family of branching function systems, however, there are many more cases with a single periodic point. For example, we have

$$
\left|B_{\infty}\left(7 a, 1+7 b, 2+7 c, 3+7 d, 4+7^{i}, 5,6\right)\right|=1
$$

for $a \in\{1,2,3,4,5\}, b \in\{0,1,2,3,4\}, c \in\{0,1,2,3\}, d \in\{0,1,2\}$ and $i=1, \ldots, 8$. Similarly,

$$
\begin{array}{rlrl}
\left|B_{\infty}\left(49,1,2,3,4+7^{i}, 5,6\right)\right| & =1, & & \left|B_{\infty}\left(56,1,2,3,4+7^{i}, 5,6\right)\right|=1, \\
\left|B_{\infty}\left(7,43,2,3,4+7^{i}, 5,6\right)\right|=1, & & \left|B_{\infty}\left(7,50,2,3,4+7^{i}, 5,6\right)\right|=1, \\
\left|B_{\infty}\left(7,1,37,3,4+7^{i}, 5,6\right)\right|=1, & & \left|B_{\infty}\left(7,1,45,3,4+7^{i}, 5,6\right)\right|=1, \\
\left|B_{\infty}\left(7,1,2,31,4+7^{i}, 5,6\right)\right|=1, & & \left|B_{\infty}\left(7,1,2,38,4+7^{i}, 5,6\right)\right|=1
\end{array}
$$

hold for $i=1, \ldots, 8$, and we conjecture that all of these equalities are valid for every positive integer $i$.

Example 5.3. Applying Theorem 5.1 for $b=r=0$ we get

$$
\left|B_{\infty}\left(n^{k}, 1, \ldots, n-1\right)\right|=\left|B_{\infty}\left(1, \ldots, n-1, n^{k}\right)\right|=1,
$$

while for $b=r=n-2$ we obtain (also taking Fact 2.2 into account)

$$
\begin{aligned}
\left|B_{\infty}\left(n-2+n^{k}, n-1, \ldots, 2 n-3\right)\right| & =\left|B_{\infty}\left(n-1, \ldots, 2 n-3, n-2+n^{k}\right)\right| \\
& =\left|B_{\infty}\left(0, \ldots, n-2, n^{k}-1\right)\right|=2^{k} .
\end{aligned}
$$

These examples show that there may be a significant difference between the cardinalities $\left|B_{\infty}\left(d_{1}, d_{2}, \ldots, d_{n}\right)\right|$ and $\left|B_{\infty}\left(d_{1}+1, d_{2}+1, \ldots, d_{n}+1\right)\right|$, at least in certain special cases. (We will see in Theorem 6.4 that for fixed $d_{1}, \ldots, d_{n-1}$ we have $\left|B_{\infty}\left(d_{1}, d_{2}, \ldots, d_{n}\right)\right|=O\left(d_{n}^{\log _{n} 2}\right)$, which yields $\left|B_{\infty}\left(0, \ldots, n-2, n^{k}-1\right)\right|=O\left(2^{k}\right)$. Therefore, we can say that the difference between $\left|B_{\infty}\left(d_{1}, d_{2}, \ldots, d_{n}\right)\right|$ and $\left|B_{\infty}\left(d_{1}+1, d_{2}+1, \ldots, d_{n}+1\right)\right|$ cannot be much larger compared to $d_{n}$ than in this example.) 
We know that $\left|B_{\infty}(0,1, \ldots, n-1)\right|=2$ and $\left|B_{\infty}\left(0,1, \ldots, n-2, n-1+n^{k}\right)\right|=$ $2^{k}+1 \geq 3$. The following proposition generalizes these results by giving a lower estimate for $\left|B_{\infty}\left(0,1, \ldots, n-2, d_{n}\right)\right|$.

Proposition 5.4. If $d_{n} \equiv n-1(\bmod n)$ and $d_{n}>n-1$, then we have

$$
\left|B_{\infty}\left(0,1, \ldots, n-2, d_{n}\right)\right| \geq 3 \text {. }
$$

If, in addition, $n-1 \mid d_{n}$, then $\left|B_{\infty}\left(0,1, \ldots, n-2, d_{n}\right)\right| \geq 4$.

Proof. Assume first that $n-1 \nmid d_{n}$. Clearly, $0 \in B_{\infty}\left(0,1, \ldots, n-2, d_{n}\right)$ and this is the only periodic point with cycle length 1 . The set $\mathcal{A} \backslash\{0\}$ is easily seen to be nonempty and closed under $R$, hence it contains a cycle, which must have length at least 2 . Thus, we have at least two more periodic points besides 0 .

If $n-1 \mid d_{n}$, then we have two periodic points with cycle length 1 , namely 0 and $-d_{n} /(n-1)$. Now $\mathcal{A} \backslash\left\{0,-d_{n} /(n-1)\right\}$ is closed under $R$, and it is nonempty, since $d_{n}>n-1$. Therefore, we have at least two periodic points in this set, hence $\left|B_{\infty}\left(0,1, \ldots, n-2, d_{n}\right)\right| \geq 4$ in this case.

Remark 5.5. The estimates given in the previous proposition are sharp: for example $B_{\infty}(0,1,32)=\{-16,-12,-4,0\}$ and $B_{\infty}(0,1,1181)=\{-443,-148,0\}$.

\section{Asymptotic Behaviour of the number of PERIOdic Points}

In this section we are interested in the number of periodic points when one or all of $d_{1}, \ldots, d_{n}$ tend to infinity. If we let all the $d_{i}$ 's go to infinity in such a way that their differences stay the same, then we obtain the sequence $\left|B_{\infty}\left(d_{1}+s, \ldots, d_{n}+s\right)\right|$ for the number of periodic points. The asymptotic behavior of this sequence as $s \rightarrow \infty$ is not very interesting, since it is periodic with period at most $n-1$ by Fact 2.2 .

$$
\left|B_{\infty}\left(d_{1}+n-1, \ldots, d_{n}+n-1\right)\right|=\left|B_{\infty}\left(d_{1}, \ldots, d_{n}\right)\right| .
$$

In some cases this sequence is constant, for instance we obtain the sequence $4,4, \ldots$ for $\left(d_{1}, d_{2}, d_{3}\right)=(0,1,17)$ and also for $\left(d_{1}, d_{2}, d_{3}\right)=(0,1,257)$. In some other cases, there might be big oscillations, as we have seen in Example 5.3. For example, we obtain the sequence $16,1,16,1, \ldots$ for $\left(d_{1}, d_{2}, d_{3}\right)=(0,1,80)$, and we get $16,1,1,16,1,1, \ldots$ for $\left(d_{1}, d_{2}, d_{3}, d_{4}\right)=(0,1,2,255)$.

Next we consider the case when $d_{1}, \ldots, d_{n}$ tend to infinity in such a way that their quotients stay the same; as before, we assume that $d_{1}<\cdots<d_{n}$. We shall see that the number of periodic points increases linearly in this case (see Theorem 6.1 in the special case $c=0)$. The proof is based on the fact that $B_{\infty}=-\mathbb{T} \cap \mathbb{Z}$ (see Proposition 1.1, where $\mathbb{T}=\mathbb{T}(D)$ is the set defined by 1.2 . Observe that the least element of $\mathbb{T}$ is $\sum_{i=1}^{\infty} n^{-i} d_{1}=\frac{d_{1}}{n-1}$; similarly, the greatest element of $\mathbb{T}$ is $\frac{d_{n}}{n-1}$, hence $\mathbb{T} \subseteq\left[\frac{d_{1}}{n-1}, \frac{d_{n}}{n-1}\right]=-\mathcal{I}$. Recall from Subection 1.2 that $\mathbb{T}$ is Jordan measurable and has measure $\mu(\mathbb{T})=\operatorname{gcd}\left\{d_{i}-d_{j}: 1 \leq i, j \leq n\right\}$.

Theorem 6.1. Let $d_{1}<\cdots<d_{n}$ be, let $c$ be an arbitrary integer and let $s \rightarrow \infty$ through integers relatively prime to $n$. Then we have

$$
\frac{\left|B_{\infty}\left(d_{1} s+c, \ldots, d_{n} s+c\right)\right|}{\left|\mathcal{A}\left(d_{1} s+c, \ldots, d_{n} s+c\right)\right|} \rightarrow \frac{n-1}{d_{n}-d_{1}} \mu(\mathbb{T}),
$$

where $\mu(\mathbb{T})$ is the Lebesgue measure of $\mathbb{T}\left(d_{1}, \ldots, d_{n}\right)$.

Proof. Let $\mathbb{T}=\mathbb{T}(D)$, and let us observe that $\mathbb{T}\left(d_{1} s+c, \ldots, d_{n} s+c\right)=s \mathbb{T}+\frac{c}{n-1}$. Therefore we have

$$
\begin{aligned}
B_{\infty}\left(d_{1} s+c, \ldots, d_{n} s+c\right) & =\left\{x \in \mathbb{Z}:-x \in \mathbb{T}\left(d_{1} s+c, \ldots, d_{n} s+c\right)\right\} \\
& =\left\{x \in \mathbb{Z}: \frac{-x-\frac{c}{n-1}}{s} \in \mathbb{T}\right\}
\end{aligned}
$$


which means that the elements of $B_{\infty}\left(d_{1} s+c, \ldots, d_{n} s+c\right)$ are in a one-to-one correspondence with the elements of the intersection of $\mathbb{T}$ and $\frac{1}{s}\left(\mathbb{Z}-\frac{c}{n-1}\right)$. Since the latter set partitions the real line into intervals of length $\frac{1}{s}$, we see that

$$
\left|B_{\infty}\left(d_{1} s+c, \ldots, d_{n} s+c\right)\right| \cdot \frac{1}{s}=\left|\mathbb{T} \cap \frac{1}{s}\left(\mathbb{Z}-\frac{c}{n-1}\right)\right| \cdot \frac{1}{s}
$$

is a Riemann sum of the characteristic function of $\mathbb{T}$. Since $\mathbb{T}$ is Jordan measurable, its characteristic function is Riemann-integrable. It follows that $\left|B_{\infty}\left(d_{1} s+c, \ldots, d_{n} s+c\right)\right|$. $\frac{1}{s} \rightarrow \mu(\mathbb{T})$ as $s \rightarrow \infty$, i.e., $\left|B_{\infty}\left(d_{1} s+c, \ldots, d_{n} s+c\right)\right|$ is asymptotically equivalent to $s \cdot \mu(\mathbb{T})$.

Since $\mathcal{A}\left(d_{1} s+c, \ldots, d_{n} s+c\right)$ consists of the integers in the interval

$$
\left[-\frac{d_{n} s+c}{n-1},-\frac{d_{1} s+c}{n-1}\right]=s \mathcal{I}-\frac{c}{n-1},
$$

the cardinality of $\mathcal{A}\left(d_{1} s+c, \ldots, d_{n} s+c\right)$ and $\frac{\left(d_{n}-d_{1}\right) s}{n-1}$ (which is the length of the interval $\left.s \mathcal{I}-\frac{c}{n-1}\right)$ differ by at most one. As a consequence, we have that

$$
\begin{gathered}
\lim _{s \rightarrow \infty} \frac{\left|B_{\infty}\left(d_{1} s+c, \ldots, d_{n} s+c\right)\right|}{\left|\mathcal{A}\left(d_{1} s+c, \ldots, d_{n} s+c\right)\right|}= \\
=\lim _{s \rightarrow \infty}\left|B_{\infty}\left(d_{1} s+c, \ldots, d_{n} s+c\right)\right| \frac{n-1}{\left(d_{n}-d_{1}\right) s}=\frac{n-1}{d_{n}-d_{1}} \mu(\mathbb{T}) .
\end{gathered}
$$

Our next goal is to study the number of periodic points when only one of the parameters, say $d_{n}$, tends to infinity, while the others are fixed. As a lower estimate we have the trivial inequality $\left|B_{\infty}(D)\right| \geq 1$, and in general we cannot have a nontrivial lower bound, since it is possible to let $d_{n} \rightarrow \infty$ in such a way that the number of periodic points stays constant 1 (cf. Theorem 5.1).

To establish an upper estimate, we will need the box-counting dimension of certain fractals. We briefly recall the necessary definitions and facts; for more background we refer the reader to $[9$. Let $\mathcal{K}$ be a bounded subset of the real line. For $\delta>0$, let $N_{\delta}(\mathcal{K})$ be the number of intervals of the form $[k \delta,(k+1) \delta)$ with $k \in \mathbb{Z}$ that contain at least one point from $\mathcal{K}$. The box-counting dimension (or Minkowski dimension) of $\mathcal{K}$ is defined as the limit

$$
\lim _{\delta \rightarrow 0} \frac{\log N_{\delta}(\mathcal{K})}{\log (1 / \delta)}
$$

provided it exists.

Let $\mathcal{C}_{n}$ be the set defined similarly to the Cantor set, successively removing the middle $\frac{n-2}{n}$ part of the intervals ( $n=3$ gives the usual Cantor set). A real number $c \in[0,1]$ belongs to $\mathcal{C}_{n}$ if and only if the base- $n$ expansion of $c$ contains only digits 0 and $n-1$. Just like the Cantor set, $\mathcal{C}_{n}$ is a self-similar fractal, and it has box-counting dimension $\log _{n} 2$. Thus we have $N_{\delta}\left(\mathcal{C}_{n}\right) \leq(1 / \delta)^{\varepsilon+\log _{n} 2}$ for small enough $\delta$; however, for our purposes the following stronger estimate will be necessary.

Proposition 6.2. For all $0<\delta<1$ we have

$$
N_{\delta}\left(\mathcal{C}_{n}\right) \leq 4 \cdot(1 / \delta)^{\log _{n} 2} .
$$

Proof. It is easy to verify that $N_{1 / n^{t}}\left(\mathcal{C}_{n}\right)=2^{t}$ for all natural numbers $t$. Now let $\delta<1$ be a positive real number, and let $t \in \mathbb{N}$ such that $1 / n^{t} \leq \delta \leq 1 / n^{t-1}$. Then we have $N_{\delta}\left(\mathcal{C}_{n}\right) \leq 2 \cdot N_{1 / n^{t}}\left(\mathcal{C}_{n}\right)$, as any interval of length $1 / n^{t}$ is covered by (at most) two intervals of length $\delta$. Taking into account that $t \leq 1+\log _{n}(1 / \delta)$, we obtain the desired inequality:

$$
N_{\delta}\left(\mathcal{C}_{n}\right) \leq 2 \cdot N_{1 / n^{t}}\left(\mathcal{C}_{n}\right)=2^{t+1} \leq 2^{2+\log _{n}(1 / \delta)}=4 \cdot(1 / \delta)^{\log _{n} 2} .
$$


The set $\frac{1}{n-1} \cdot \mathcal{C}_{n}$ consists of all numbers of the interval $[0,1]$ (actually, they are from the interval $\left.\left[0, \frac{1}{n-1}\right]\right)$ that have only 0 and 1 in their base- $n$ representation. Obviously, $\frac{1}{n-1} \cdot \mathcal{C}_{n}$ also has box-counting dimension $\log _{n} 2$, and we have a similar estimate for $N_{\delta}$ as for $\mathcal{C}_{n}$.

Corollary 6.3. There is a positive constant $K$ depending only on $n$ such that for all $0<\delta<\frac{1}{n-1}$ we have

$$
N_{\delta}\left(\frac{1}{n-1} \cdot \mathcal{C}_{n}\right) \leq K \cdot\left(\frac{1}{\delta}\right)^{\log _{n} 2}
$$

Proof. Clearly, $N_{\delta}\left(\frac{1}{n-1} \cdot \mathcal{C}_{n}\right)=N_{(n-1) \delta}\left(\mathcal{C}_{n}\right)$, hence

$$
N_{\delta}\left(\frac{1}{n-1} \cdot \mathcal{C}_{n}\right) \leq 4 \cdot\left(\frac{1}{(n-1) \delta}\right)^{\log _{n} 2}
$$

Theorem 6.4. If $d_{1}<\cdots<d_{n-1}$ are fixed and $d_{n} \rightarrow \infty$, then

$$
\left|B_{\infty}\left(d_{1}, \ldots, d_{n}\right)\right|=O\left(d_{n}^{\log _{n} 2}\right) \text {. }
$$

Proof. Let us decompose an arbitrary $x=\sum_{i=1}^{\infty} \frac{a_{i}}{n^{i}} \in \mathbb{T}$ as $x=\eta_{x}+\gamma_{x}$, where

$$
\eta_{x}=\sum_{a_{i} \neq d_{n}} \frac{a_{i}}{n^{i}} \text { and } \gamma_{x}=\sum_{a_{i}=d_{n}} \frac{a_{i}}{n^{i}} .
$$

Then we have $0 \leq \eta_{x} \leq d_{n-1} \cdot \sum_{i=1}^{\infty} \frac{1}{n^{i}}=\frac{d_{n-1}}{n-1}$, and $\gamma_{x}=d_{n} \cdot \sum_{a_{i}=d_{n}} \frac{1}{n^{i}}=d_{n} \cdot c_{x}$, where $c_{x} \in[0,1]$ has only digits 0 and 1 in its base- $n$ expansion, i.e., $c_{x} \in \frac{1}{n-1} \cdot \mathcal{C}_{n}$. If $x$ is an integer, then it must be one of the numbers $\left\lfloor\gamma_{x}\right\rfloor,\left\lfloor\gamma_{x}\right\rfloor+1, \ldots,\left\lfloor\gamma_{x}\right\rfloor+\left\lfloor\frac{d_{n-1}}{n-1}\right\rfloor+1$, thus we obtain the following "upper estimate" for the (negative of the) set of periodic points:

$$
\begin{gathered}
-B_{\infty}(D)=\mathbb{T} \cap \mathbb{Z} \subseteq \\
\bigcup_{c \in \frac{1}{n-1} \cdot \mathcal{C}_{n}}\left\{\left\lfloor d_{n} \cdot c\right\rfloor,\left\lfloor d_{n} \cdot c\right\rfloor+1, \ldots,\left\lfloor d_{n} \cdot c\right\rfloor+\left\lfloor\frac{d_{n-1}}{n-1}\right\rfloor+1\right\} .
\end{gathered}
$$

Each set of this union has $\left\lfloor\frac{d_{n-1}}{n-1}\right\rfloor+2$ elements, and the sets corresponding to $c$ and $c^{\prime}$ coincide if and only if $\left\lfloor d_{n} \cdot c\right\rfloor=\left\lfloor d_{n} \cdot c^{\prime}\right\rfloor$, hence

$$
\left|B_{\infty}(D)\right|=|\mathbb{T} \cap \mathbb{Z}| \leq\left(\left\lfloor\frac{d_{n-1}}{n-1}\right\rfloor+2\right) \cdot\left|\left\{\left\lfloor d_{n} \cdot c\right\rfloor: c \in \frac{1}{n-1} \cdot \mathcal{C}_{n}\right\}\right| .
$$

An integer $k$ appears as $\left\lfloor d_{n} \cdot c\right\rfloor$ in the formula above if and only if there exists $c \in \frac{1}{n-1} \cdot \mathcal{C}_{n}$ such that $d_{n} \cdot c \in[k, k+1)$. The latter is equivalent to $c \in\left[\frac{k}{d_{n}}, \frac{k}{d_{n}}+\frac{1}{d_{n}}\right)$, therefore

$$
\left|B_{\infty}(D)\right|=|\mathbb{T} \cap \mathbb{Z}| \leq\left(\left\lfloor\frac{d_{n-1}}{n-1}\right\rfloor+2\right) \cdot N_{1 / d_{n}}\left(\frac{1}{n-1} \cdot \mathcal{C}_{n}\right) .
$$

From Corollary 6.3 it follows that for $d_{n}>n-1$ we have

$$
N_{1 / d_{n}}\left(\frac{1}{n-1} \cdot \mathcal{C}_{n}\right) \leq K \cdot d_{n}^{\log _{n} 2}
$$

for some constant $K$, and this together with 6.1 implies that $\left|B_{\infty}\right|=O\left(d_{n}^{\log _{n} 2}\right)$.

The next theorem shows that the upper estimate obtained above cannot be sharpened: for fixed $d_{2}, \ldots, d_{n-1}$ it is possible to let $d_{n} \rightarrow \infty$ in such a way that $\left|B_{\infty}\left(0, d_{2}, \ldots, d_{n}\right)\right|$ is bounded from below by a constant multiple of $d_{n}^{\log _{n} 2}$.

Theorem 6.5. Let $0, d_{2}, \ldots, d_{n-1}, r_{n}$ be a complete system of residues modulo $n$. Then there is a sequence $d_{n, 1}, d_{n, 2}, \ldots$ of positive integers tending to $\infty$ such that for all $\ell \in \mathbb{N}, d_{n, \ell} \equiv r_{n}(\bmod n)$, and $\left|B_{\infty}\left(0, d_{2}, \ldots, d_{n-1}, d_{n, \ell}\right)\right|=\Theta\left(d_{n, \ell}^{\log _{n} 2}\right)$ as $\ell \rightarrow \infty$. 
Proof. Let $d_{n, \ell}$ be the least positive integer such that $d_{n, \ell} \equiv r_{n}(\bmod n)$ and $d_{n, \ell} \equiv$ $0\left(\bmod n^{\ell}-1\right)$. Since the moduli are relatively prime, such a $d_{n, \ell}$ exists, and $d_{n, \ell} \leq$ $n\left(n^{\ell}-1\right)<n^{\ell+1}$. (Note that $d_{n, \ell} \geq n^{\ell}-1$, therefore $d_{n, \ell}$ indeed tends to $\infty$ as $\ell \rightarrow \infty$.) We are going to prove that there are at least $2^{\ell}$ periodic points for $0, d_{2}, \ldots, d_{n-1}, d_{n, \ell}$. This suffices to prove the theorem, since

$$
2^{\ell}=\frac{1}{2}\left(n^{\ell+1}\right)^{\log _{n} 2}>\frac{1}{2} d_{n, \ell}^{\log _{n} 2},
$$

thus the number of periodic points is bounded from below by a constant multiple of $d_{n, \ell}^{\log _{n} 2}$, and we have seen in Theorem 6.4 that it is also bounded from above by a constant multiple of $d_{n, \ell}^{\log _{n} 2}$.

Let us choose a sequence $a_{0}, \ldots, a_{\ell-1}$ such that $a_{i} \in\left\{0, d_{n, \ell}\right\}$ for $i=1, \ldots, \ell$. There are $2^{\ell}$ such sequences, and each of them gives a periodic point

$$
x=-\frac{a_{0}+a_{1} n+\cdots+a_{\ell} n^{\ell-1}}{n^{\ell}-1} .
$$

Indeed, by Remark 2.7, we only need to verify that $x$ is an integer, which is clearly the case, as $a_{i} \in\left\{0, d_{n, \ell}\right\}$ and $d_{n, \ell} \equiv 0\left(\bmod n^{\ell}-1\right)$.

\section{SOME OPEN PROBLEMS}

Concluding the paper, we list some open problems that seem worthwhile investigating.

Theorem 3.6 characterizes the equivalence of branching function systems corresponding to arithmetic sequences. Here we required that the equivalence preserves the colors of the edges of the underlying graphs, i.e., $d_{i}$ corresponds to $d_{i}^{\prime}$. It would be interesting to consider a weaker notion of equivalence, where we are allowed to permute the colors (i.e., $d_{i}$ may correspond to some $d_{j}^{\prime}$ with $j \neq i$ ). We conjecture that Theorem 3.6 remains valid in this more general setting, too. Another natural extension of Theorem 3.6 would be to classify the branching function systems arising from the "almost arithmetic" sequences of Section 4 up to equivalence.

Corollary 3.5 also raises a natural question: Does every conjugation-closed set of primitive words arise as the set of words corresponding to the periodic points of a branching function system determined by a complete system of residues $d_{1}, \ldots, d_{n}$ ? (The answer is obviously "yes" if one allows arbitrary branching function systems.)

In Section 5 we presented examples with a single periodic point; however, there are probably many other such branching function systems, and it is still an open problem to characterize these. Proposition 5.4 gives a negative result: setting $d_{1}=$ $0, \ldots, d_{n-1}=n-2$, there is no $d_{n}$ such that $\left|B_{\infty}(D)\right|=1$. Is it possible to fix $n-2$ parameters in such a way that the number of periodic points is at least 2, no matter what the remaining two parameters are?

We had to assume $d_{1}=0$ in Theorem 6.5 in order to make our construction work. Is it possible to remove this assumption?

Figure 2 shows the distribution of the pairs $\left(\frac{a}{b}, \min \left(0.5, \frac{\left|B_{\infty}\right|}{|\mathcal{A}|}\right)\right)$ for triples of the form $(0, a, b)$ where $9000<b<9300$ and $0<a<\frac{b}{2}$. (We have cut the graph at $y=0.5$ in order to get a better resolution for smaller ratios.) The data has been calculated by a $\mathrm{C}++$ program.

The graph allows us to formulate some conjectures:

- Theorem 6.1 shows that for example triples of the form $(0, a, 5 a)$ yield proportionately the same amount of periodic points for large $a$ 's. The graph shows furthermore that triples of the form $(0, a+\epsilon, 5 a+\epsilon)$ where $a$ is large and $\epsilon$ is small compared to $a$ yield considerably fewer periodic points, and they yield fewer as $\epsilon$ gets larger. This suggests that triples of the form $(0, a, b)$ yield locally the most periodic points when the greatest common divisor of $a$ and $b$ is big, and they also induce more periodic points in their neighbourhood, but 


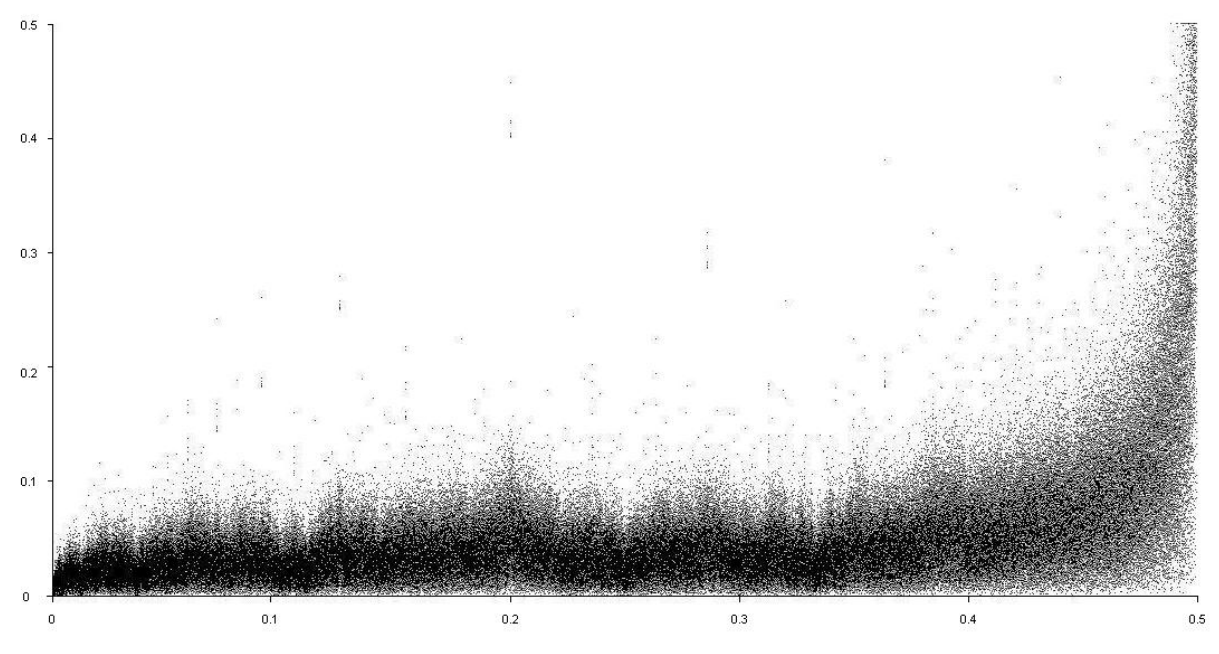

FigURE 2. A graph of points of the form $\left(\frac{a}{b}, \min \left(0.5, \frac{B_{\infty}(0, a, b)}{\mathcal{A}(0, a, b)}\right)\right)$

the radius of this influence seems to be sublinear in $b$ (the spikes on the same graph corresponding to bigger $b$ 's are narrower).

- If $\frac{a}{b}$ is close to $\frac{1}{2}$ then the ratio $\frac{\left|B_{\infty}\right|}{|\mathcal{A}|}$ is closer to 1 , however, not for all such $a$ 's. Recall that the ratio equals 1 if $a=\frac{b}{2}$ by Theorem 3.1 .

\section{ACKNOWLEDGEMENTS}

The authors would like to thank Dr. Mark Lawson for initiating this research, for his inspiring questions and for his help and hospitality during the first author's visits to Edinburgh. Helpful discussions with László Imre Szabó and Béla Nagy are also gratefully acknowledged. The first author acknowledges the support of the Engineering and Physical Sciences Research Council (EPSRC) grant no. EP/I032312/1. Research is partially supported by the Hungarian Scientific Research Fund (OTKA) grants no. K83219 and K104251, by the European Union and co-funded by the European Social Fund under the project "Telemedicine-focused research activities on the field of Mathematics, Informatics and Medical sciences" of project number "TÁMOP4.2.2.A-11/1/KONV-2012-0073". The second author is supported by the János Bolyai Research Scholarship.

\section{REFERENCES}

1. C. Bandt and Y. Wang, Disk-like self-affine tiles in $\mathbb{R}^{2}$, Discrete Comput. Geom. 26 (2001), no. 4, 591-601. MR 1863811

2. Christoph Bandt, Self-similar sets. V. Integer matrices and fractal tilings of $\mathbf{R}^{n}$, Proc. Amer. Math. Soc. 112 (1991), no. 2, 549-562. MR 1036982

3. Ievgen V. Bondarenko and Rostyslav V. Kravchenko, On Lebesgue measure of integral self-affine sets, Discrete Comput. Geom. 46 (2011), no. 2, 389-393. MR 2812515

4. Ola Bratteli and Palle E. T. Jorgensen, Iterated function systems and permutation representations of the Cuntz algebra, Mem. Amer. Math. Soc. 139 (1999), no. 663, x+89. MR 1469149

5. Joachim Cuntz, Simple $C^{*}$-algebras generated by isometries, Comm. Math. Phys. 57 (1977), no. 2, 173-185. MR 0467330

6. Dorin Ervin Dutkay and John Haussermann, Number theory problems from the harmonic analysis of a fractal, J. Number Theory 159 (2016), 7-26. MR 3412709

7. Dorin Ervin Dutkay, John Haussermann, and Palle E. T. Jorgensen, Atomic representations of Cuntz algebras, J. Math. Anal. Appl. 421 (2015), no. 1, 215-243. MR 3250475

8. P. Duvall, J. Keesling, and A. Vince, The Hausdorff dimension of the boundary of a self-similar tile, J. London Math. Soc. (2) 61 (2000), no. 3, 748-760. MR 1766102

9. Kenneth Falconer, Fractal geometry, second ed., John Wiley \& Sons, Inc., Hoboken, NJ, 2003, Mathematical foundations and applications. MR 2118797

10. William J. Gilbert, Geometry of radix representations, The geometric vein, Springer, New YorkBerlin, 1981, pp. 129-139. MR 661773 
11. _ Radix representations of quadratic fields, J. Math. Anal. Appl. 83 (1981), no. 1, 264-274. MR 632342

12. K. Gröchenig and W. R. Madych, Multiresolution analysis, Haar bases, and self-similar tilings of $\mathbf{R}^{n}$, IEEE Trans. Inform. Theory 38 (1992), no. 2, part 2, 556-568. MR 1162214

13. Karlheinz Gröchenig and Andrew Haas, Self-similar lattice tilings, J. Fourier Anal. Appl. 1 (1994), no. 2, 131-170. MR 1348740

14. Derek Hacon, Nicolau C. Saldanha, and J. J. P. Veerman, Remarks on self-affine tilings, Experiment. Math. 3 (1994), no. 4, 317-327. MR 1341723

15. Eui-Chai Jeong, Irreducible representations of the Cuntz algebra $\mathcal{O}_{N}$, Proc. Amer. Math. Soc. 127 (1999), no. 12, 3583-3590. MR 1621953

16. D. G. Jones and M. V. Lawson, Strong representations of the polycyclic inverse monoids: cycles and atoms, Period. Math. Hungar. 64 (2012), no. 1, 53-87. MR 2886407

17. Mark Kambites, Formal languages and groups as memory, Comm. Algebra 37 (2009), no. 1, 193-208. MR 2482816

18. I. Kátai and J. Szabó, Canonical number systems for complex integers, Acta Sci. Math. (Szeged) 37 (1975), no. 3-4, 255-260. MR 0389759

19. Katsunori Kawamura, Yoshiki Hayashi, and Dan Lascu, Continued fraction expansions and permutative representations of the Cuntz algebra $\mathcal{O}_{\infty}$, J. Number Theory 129 (2009), no. 12, 30693080. MR 2560855

20. James Keesling, The boundaries of self-similar tiles in $\mathbf{R}^{n}$, Topology Appl. 94 (1999), no. 1-3, 195-205, Special issue in memory of B. J. Ball. MR 1695356

21. Jeffrey C. Lagarias and Yang Wang, Haar bases for $L^{2}\left(\mathbf{R}^{n}\right)$ and algebraic number theory, J. Number Theory 57 (1996), no. 1, 181-197. MR 1378581

22. __ Integral self-affine tiles in $\mathbf{R}^{n}$. I. Standard and nonstandard digit sets, J. London Math. Soc. (2) 54 (1996), no. 1, 161-179. MR 1395075

23. _ Self-affine tiles in $\mathbf{R}^{n}$, Adv. Math. 121 (1996), no. 1, 21-49. MR 1399601

24. _ Integral self-affine tiles in $\mathbf{R}^{n}$. II. Lattice tilings, J. Fourier Anal. Appl. 3 (1997), no. 1, 83-102. MR 1428817

25. Mark V. Lawson, Orthogonal completions of the polycyclic monoids, Comm. Algebra 35 (2007), no. 5, 1651-1660. MR 2317635

26. _ The polycyclic monoids $P_{n}$ and the Thompson groups $V_{n, 1}$, Comm. Algebra 35 (2007), no. 12, 4068-4087. MR 2372319

27. _ Primitive partial permutation representations of the polycyclic monoids and branching function systems, Period. Math. Hungar. 58 (2009), no. 2, 189-207. MR 2531164

28. John Meakin and Mark Sapir, Congruences on free monoids and submonoids of polycyclic monoids, J. Austral. Math. Soc. Ser. A 54 (1993), no. 2, 236-253. MR 1200795

29. Maurice Nivat and Jean-François Perrot, Une généralisation du monoïde bicyclique, C. R. Acad. Sci. Paris Sér. A-B 271 (1970), A824-A827. MR 0271258

30. Andrew Vince, Digit tiling of Euclidean space, Directions in mathematical quasicrystals, CRM Monogr. Ser., vol. 13, Amer. Math. Soc., Providence, RI, 2000, pp. 329-370. MR 1798999

(M. Hartmann) Bolyai Institute, University of Szeged, Aradi vértanúk tere 1, H-6720 Szeged, Hungary

E-mail address: hartm@math.u-szeged.hu

(T. Waldhauser) Bolyai Institute, University of Szeged, Aradi vértanúk tere 1, H-6720 Szeged, Hungary

E-mail address: twaldha@math.u-szeged.hu 